\title{
HOPF ALGEBRAS UP TO HOMOTOPY
}

\author{
DAVID J. ANICK
}

\section{BACKGROUND AND SUMMARY}

Ever since functors from the category TOP of topological spaces to various algebraic categories were first discovered, topologists have sought functors which are complete homotopy invariants. A functor is called a "homotopy invariant" if its values on two maps $f$ and $g$ coincide (or are equivalent in some nice enough sense) whenever $f$ and $g$ are homotopic. For a "complete homotopy invariant" one requires the converse as well. Having a complete homotopy invariant on a subcategory $\mathscr{C}$ of TOP means that one can study all the homotopy-theoretic properties of $\mathscr{C}$ purely by doing algebra.

The single most exploited complete homotopy invariant has been the Quillen model $^{1}$ for Q-local simply-connected spaces [13]. This model associates to each such space a differential graded Lie algebra over $\mathbf{Q}$. A "differential graded Lie algebra" (henceforth "dgL") over $\mathbf{Q}$ is a positively graded $\mathbf{Q}$-module, $L=$ $\bigoplus_{n=-\infty}^{\infty} L_{n}$ with $L_{n}=0$ for $n \leq 0$, together with a bilinear bracket [, ]: $L_{m} \otimes$ $L_{n} \rightarrow L_{m+n}$ and a differential $\delta: L_{n} \rightarrow L_{n-1}$. The bracket must satisfy the Jacobi identities (with signs) for a Lie algebra, and $\delta$ is a derivation whose square is zero. There is a reasonable concept of "homotopy" among homomorphisms between two dgL's, analogous in many respects to the topological notion of homotopy. Using this concept, Quillen showed that rational homotopy types of simply-connected spaces biject naturally with homotopy types of dgL's, and likewise for maps. This seminal discovery has made possible the immensely successful field of rational homotopy theory.

An older, noncomplete homotopy invariant was discovered by Adams and Hilton [2]. For each simply-connected CW complex their construction yields an associative differential graded algebra (henceforth "dga"). Compared with Quillen's model, the Adams-Hilton model has the advantages of being easier to compute in practice, and of providing information over an arbitrary ring $R$ (not just $\mathbf{Q}$ ). Quillen's model enjoys the comparative strengths of being a Lie rather than an associative algebra, meaning that it contains less superfluous

Received by the editors February 23, 1988 and, in revised form, December 12, 1988.

1980 Mathematics Subject Classification (1985 Revision). Primary 55P62, 18 G55.

The author was partially supported by a National Science Foundation grant.

${ }^{1} \mathrm{~A}$ "model" is for practical purposes a functor, but uniqueness and compatibility with compositions may hold only up to homotopy. 
information, and as we have mentioned, it is rationally a complete homotopy invariant.

Let $R$ denote a subring of $\mathbf{Q}$. In [6] and [7], Baues, Halperin, and Lemaire established a compatibility between the Adams-Hilton (over $R$ ) and the Quillen models. For spaces of finite type, the $\mathbf{Q}$-localization of Adams-Hilton coincides (up to homotopy) with the universal enveloping algebra (denoted $U$ ) of Quillen. This is illustrated by the solid arrows in (1).

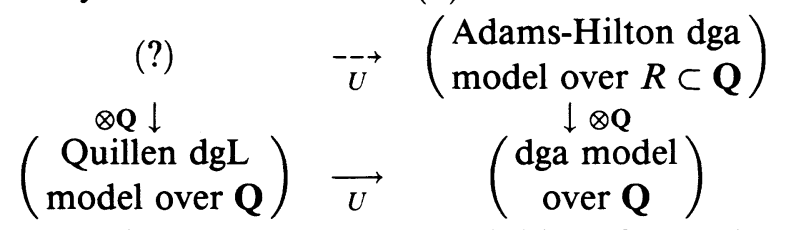

The dotted arrows in (1) suggest the possibility of a "unified model," represented by the question mark, enjoying the strong points of both models. It is here that this paper comes in. Under certain dimension and connectivity conditions, we construct precisely such a unified model. Since we adopt the Adams-Hilton model as our starting point, one consequence is a new construction of the Quillen model.

Specifically, if for some $p R$ contains $n^{-1}$ for $n<p$, and if we restrict ourselves for some $r \geq 1$ to $r$-connected $\mathrm{CW}$ complexes of dimension $\leq r p$, then (1) may be filled in. The simple fact that the Adams-Hilton model for $X$ is an enveloping algebra under these hypotheses also leads to a quick proof of Wilkerson's conjecture, viz., the vanishing of $p$ th powers in $\widetilde{H}^{*}\left(\Omega X ; \mathbf{Z}_{p}\right)$.

The bulk of this paper will be dedicated to the proof of a single purely algebraic theorem, Theorem 4.8. This theorem asserts that certain dga's are automatically the enveloping algebras of dgL's. We will now describe how these results were discovered, hoping thereby to elucidate the key ideas involved as well as what we mean by "certain dga's."

Consider a typical dga, $(A, d)$. Is $(A, d)$ the Adams-Hilton model for any space? In general, the answer will be "no." What properties distinguish dga's which are Adams-Hilton models from those which are not?

Here is a dga which is not an Adams-Hilton model: $A=R\langle x, y\rangle=$ free associative $R$-algebra on $x$ and $y,|x|=1,|y|=4, d(x)=0, d(y)=x^{3}$. Suppose $(A, d)$ were the Adams-Hilton model for some $X$, denoted $\mathbf{A}(X)$. Consider the diagonal map $\Delta: X \rightarrow X \times X$, and the induced homomorphism

$$
\mathbf{A}(X) \stackrel{\mathbf{A}(\Delta)}{\longrightarrow} \mathbf{A}(X \times X) \stackrel{\simeq}{\underset{j}{\longrightarrow}} \mathbf{A}(X) \otimes \mathbf{A}(X) .
$$

(We must borrow here the quasi-isomorphism $j$ from the original AdamsHilton paper [2, p. 322].) Denote by $\psi$ this composite dga homomorphism. Then $\psi(x)=x \otimes 1+1 \otimes x$, so

$$
d \psi(y)=\psi d(y)=\psi\left(x^{3}\right)=\psi(x)^{3}=x^{3} \otimes 1+x^{2} \otimes x+x \otimes x^{2}+1 \otimes x^{3} .
$$

But $x^{3} \otimes 1+x^{2} \otimes x+x \otimes x^{2}+1 \otimes x^{3}$ is not a boundary in $(A, d) \otimes(A, d)$, so $\psi(y)$ cannot exist! 
In order for a dga $(A, d)$ to equal $\mathbf{A}(X)$, it must be capable of receiving a "coproduct" $\psi:(A, d) \rightarrow(A, d) \otimes(A, d)$. Since this coproduct is to come from a model for the diagonal map, some of its properties are constrained. As we shall see in $\S 8$, there must be homotopies between $\left(1_{A} \otimes \varepsilon\right) \psi$ and $1_{A}$, between $\left(\varepsilon \otimes 1_{A}\right) \psi$ and $1_{A}$, between $\tau \psi$ and $\psi$, and between $(\psi \otimes 1) \psi$ and $(1 \otimes \psi) \psi$. Here $\varepsilon: A \rightarrow R$ is the augmentation, $1_{A}: A \rightarrow A$ is the identity, and $\tau$ sends $a \otimes b$ to $(-1)^{|a||b|} b \otimes a$. The idea is to consider only those $(A, d)$ for which there exists a $\psi$ permitting these four homotopies.

If we had equalities in place of the four homotopies, $\psi$ would make $(A, d)$ into a differential graded cocommutative coassociative Hopf algebra. As it stands, we call the triple $(A, d, \psi)$ a "Hopf algebra up to homotopy" or "Hah." Our point of view becomes the following. Keeping the diagonal information (2), we view the Adams-Hilton model as converting spaces not into dga's, but into Hopf algebras up to homotopy.

Recall that the universal enveloping algebra of a graded Lie algebra is automatically a cocommutative coassociative Hopf algebra. For a $\operatorname{dgL}(L, \delta)$, $U(L, \delta)$ is automatically a differential graded Hopf algebra, which is a special kind of Hah. A remarkable converse also holds: within certain connectivity and dimension constraints, every $\mathrm{Hah}$ is isomorphic to $U(L, \delta)$ for some (unique) $(L, \delta)$ ! It follows for suitable spaces $X$ that $\mathbf{A}(X) \approx U(L, \delta)$. We may now adopt $(L, \delta)$ as our Lie algebra model for $X$, thus filling in diagram (1).

Theorem 4.8 is, of course, precisely this "remarkable converse." We are at last in a position to state it precisely. Let $R$ contain $n^{-1}$ for $n<p$. Given a Hah $(A, d, \psi)$, where $A$ is a tensor algebra generated by the range $A_{r}$ through $A_{r p-1}$, there is a $\operatorname{dgL}(L, \delta)$ for which $U(L, \delta) \approx(A, d)$, and the Hopf algebra coproduct $\Delta_{L}$ on $U(L, \delta)$ is homotopic with $\psi$. The homotopy type of $(L, \delta)$ is determined by that of $(A, d, \psi)$. Furthermore, for any homomorphism $f:(A, d, \psi) \rightarrow\left(A^{\prime}, d^{\prime}, \psi^{\prime}\right)$ satisfying $\psi^{\prime} f \simeq(f \otimes f) \psi$, there is an $\alpha:(L, \delta) \rightarrow\left(L^{\prime}, \delta^{\prime}\right)$ such that $U \alpha \simeq f$ once we identify $(A, d)$ with $U(L, \delta)$ and $\left(A^{\prime}, d^{\prime}\right)$ with $U\left(L^{\prime}, \delta^{\prime}\right)$. Lastly, the homotopy class of $\alpha$ is determined by the class of $f$ (cf. [4]).

Here is the briefest overview of the proof of Theorem 4.8. First, consider the "nondifferential" version, essentially due to Milnor and Moore [10], that a cocommutative coassociative free Hopf $R$-algebra generated in the stated range is primitively generated (hence an enveloping algebra). We can give a proof of this nondifferential version via splittings of a certain exact sequence. Inducting on the dimension, if the reduced coproduct of some generator is nonzero, it is possible to subtract from it a decomposable element so that the difference, also a valid choice for the generator, is primitive.

The differential version works much the same way. Assuming we have constructed a strictly cocommutative coassociative $\chi$ homotopic to $\psi$ in dimensions below $n$, we utilize the four homotopies together with the splittings to adjust $\chi$, so it becomes strictly cocommutative and coassociative in dimension 
$n$ as well. This procedure requires a detailed understanding of the homotopy theory for the category of dga's, as well as some delicate properties of the splittings.

Finally, let us mention some new open questions which arise from this work. Is a free Hah always isomorphic to a differential graded (not necessarily primitively generated) Hopf algebra? Can our model be improved by incorporating into it the action of the Steenrod algebra? Which theorems of rational homotopy remain valid over subrings of $\mathbf{Q}$ or over $\mathbf{Z}_{p}$, subject to dimension and connectivity hypotheses?

\section{HOMOTOPY THEORY FOR DIFFERENTIAL GRADED ALGEBRAS}

We collect in this section all the definitions and lemmas we shall use later about the category DGA of differential graded algebras. Since none of the results are new, their proofs have been omitted or drastically curtailed. Still, the material is presented in sequence, so what follows is actually a development of the concept of homotopy in DGA, minus the proofs. References include [3, $7,11]$.

Definitions. Let $R$ denote a commutative ring with unity. A graded algebra over $R$ is a graded $R$-module $A=\bigoplus_{n=-\infty}^{\infty} A_{n}, A_{n}=0$ for $n<0$, with associative bilinear pairings $A_{m} \otimes A_{n} \rightarrow A_{m+n}$. It is connected if $1 \in A_{0} \approx R$. It is r-reduced if it is connected and $A_{n}=0$ for $0<n<r$. Connected algebras have a unique graded algebra homomorphism $\varepsilon: A \rightarrow R$ called the augmentation. $A_{+}=\operatorname{ker}(\varepsilon)$ is the augmentation ideal. For $x \in A_{n}, x \neq 0$, we write $|x|=n$ and call $n$ the dimension of $x$.

A differential on a graded algebra $A$ is an $R$-homomorphism $d$ of degree -1 satisfying $d^{2}=0$ and

$$
d(x y)=d(x) y+(-1)^{|x|} x d(y)
$$

A differential graded algebra (henceforth $d g a$ ) is a connected graded algebra $A$ together with a differential $d$ for which $d\left(A_{+}\right) \subseteq A_{+}$. Our notation is $(A, d)$ or, where no confusion can result, simply $A$.

A homomorphism of dga's is both a graded algebra homomorphism and a chain map. The category of dga's over $R$ is denoted $\operatorname{DGA}(R)$, or simply DGA. Homology, denoted $H_{*}()$, is a functor from DGA to connected graded algebras. A DGA homomorphism inducing an isomorphism on homology is called a quasi-isomorphism or quism.

The category of connected graded algebras has push-outs. The push-out of the diagram $B \stackrel{f}{\leftarrow} A \stackrel{g}{\rightarrow} C$ is denoted $B \amalg_{A} C$. Most often, we consider pushouts over the trivial graded algebra $R$, for which the notation is simply $B \amalg C$. As an $R$-module, $B \amalg C$ equals the direct sum of the graded $R$-modules

$$
R, B_{+}, C_{+}, B_{+} \otimes C_{+}, C_{+} \otimes B_{+}, B_{+} \otimes C_{+} \otimes B_{+}, \ldots
$$


It has the obvious multiplicative structure. If $f$ and $g$ are DGA homomorphisms, then $B \amalg_{A} C$, endowed with the obvious differential, is the push-out in DGA of the diagram $(B, e) \stackrel{f}{\leftarrow}(A, d) \stackrel{g}{\rightarrow}(C, \gamma)$.

The tensor product of dga's $(A, d)$ and $(B, e)$ is $(A \otimes B, \delta)$, where $\delta(a \otimes b)=$ $d(a) \otimes b+(-1)^{|a|} a \otimes e(b)$. Their direct product, denoted $(A, d) \times(B, e)$ or $A \times B$, is given by $(A \times B)_{0}=R,(A \times B)_{n}=A_{n} \oplus B_{n}$ for $n>0$, with componentwise addition, multiplication, and differentiation. There are obvious DGA surjections $A \amalg B \rightarrow A \otimes B \rightarrow A \times B \rightrightarrows A, B$.

Given a free positively graded $R$-module $V, T V$ denotes the tensor algebra on $V$ with grading inherited from $V$. We identify $V$ with $T^{1} V \subseteq T V$. We call a dga $(A, d)$ free if $A$ is isomorphic with some $T V$. Note that $A$ need not be a free object in DGA. Given a graded set $S$ (i.e., $S$ together with $\left.e: S \rightarrow \mathrm{Z}_{+}\right)$the free associative algebra on $S$ is denoted $R\langle S\rangle$. Clearly, $R\langle S\rangle$ and $T\left(\operatorname{Span}_{R}(S)\right)$ coincide.

Lemma 2.1. Let $f:(A, d) \rightarrow(B, e)$ be a DGA homomorphism.

(a) $f$ may be factored as

$$
(A, d) \stackrel{h}{\rightarrow}(A, d) \amalg(T V, \delta) \stackrel{p}{\rightarrow}(B, e),
$$

where $h$ is a quism and $p$ is surjective.

(b) $f$ may be factored as

$$
(A, d) \stackrel{j}{\rightarrow}(A \amalg T W, \delta) \stackrel{q}{\rightarrow}(B, e),
$$

where $q$ is a quism.

Definition. Let $(A, d)$ be a dga. A dga $\left(A^{*}, d^{*}\right)$ together with homomorphisms

$$
(A, d) \underset{j_{2}}{\stackrel{j_{1}}{\rightrightarrows}}\left(A^{*}, d^{*}\right) \stackrel{p}{\rightarrow}(A, d)
$$

is called a cylinder on $(A, d)$ if

(i) $j_{1} \amalg j_{2}:(A, d) \amalg(A, d) \rightarrow\left(A^{*}, d^{*}\right)$ is injective;

(ii) $p j_{1}=p j_{2}=1_{A}$; and

(iii) $p$ is a quism.

We say that two homomorphisms $f, g:(A, d) \rightarrow(B, e)$ are homotopic, $f \simeq g$, if $f \amalg g:(A, d) \amalg(A, d) \rightarrow(B, e)$ extends over some cylinder $\left(A^{*}, d^{*}\right)$.

By Lemma 2.1(b) applied to the collapse map $A \amalg A \rightarrow A$, every dga has a cylinder.

Lemma 2.2. (a) If $A \stackrel{h}{\rightarrow} B \underset{g}{\stackrel{f}{\rightarrow}} C \stackrel{i}{\rightarrow} D$ are $D G A$ homomorphisms and $f \simeq g$, then ifh $\simeq$ igh.

(b) $\simeq$ is an equivalence relation.

Remark. Only transitivity presents any challenge. It follows from the observation that the push-out over $A$ of two cylinders on $A$ is another cylinder. 
When $A$ is free, we can give an alternate criterion for two homomorphisms with source $A$ to be homotopic.

Definition. Let $f, g:(A, d) \rightarrow(B, e)$. A degree $r R$-homomorphism $F: A \rightarrow$ $B$ is an $(f, g)$-derivation if it satisfies

$$
F(x y)=F(x) g(y)+(-1)^{r|x|} f(x) F(y) .
$$

A derivation homotopy from $f$ to $g$ is an $(f, g)$-derivation of degree +1 further satisfying

$$
e F+F d=f-g .
$$

Lemma 2.3. Let $f, g: T V \rightarrow B$ be algebra homomorphisms and let $F: V \rightarrow B$ be $R$-linear of degree $r$. Then $F$ extends uniquely to an $(f, g)$-derivation $\widetilde{F}: T V \rightarrow B$. If in addition $f$ and $g$ are DGA homomorphisms and $\left.\widetilde{F}\right|_{V}$ satisfies (4), then $\widetilde{F}$ satisfies (4).

Remark. The proof of Lemma 2.3 does not require that $d^{2}=0$ or $e^{2}=0$.

In [7], Baues and Lemaire construct a canonical cylinder on a free dga $(A, d)$. We denote this the Baues-Lemaire cylinder by $I(A, d)$ or $(I A, D)$, and we recall briefly its construction. If $A=T V$, then $I A=T\left(V \oplus V^{\prime} \oplus S V\right)$ as an algebra, where $V^{\prime} \approx V$ and $(s V)_{n} \approx(V)_{n-1}$. To define $D$, first put $f, f^{\prime}: T V \rightarrow$ $I A$ by $f(x)=x$ and $f^{\prime}(x)=x^{\prime}$. Using Lemma 2.3 , let $S$ be the unique $\left(f, f^{\prime}\right)$-derivation having $S(x)=s x$ for $x \in V$. Setting $D(x)=f d(x)$ and $D\left(x^{\prime}\right)=f^{\prime} d(x)$ and $D(s x)=x-x^{\prime}-S d(x)$, extend $D$ (uniquely) to a $(1,1)-$ derivation of degree -1 on $I A$. Then $S d+D S$ is an $\left(f, f^{\prime}\right)$-derivation. Hence by Lemma 2.3 ,

$$
S d+D S=f-f^{\prime}
$$

because this holds on $V$, even though we do not know yet that $D^{2}=0$. Using (5), now check that $D^{2}$ is a $(1,1)$-derivation of degree -2 , vanishing on each generator of $I A$, so $D^{2}=0$. We have constructed the dga $(I A, D)$.

Lemma 2.4. The homomorphisms $j_{1}(x)=x, j_{2}(x)=x^{\prime}$, and $p(x)=p\left(x^{\prime}\right)=$ $x, p(s x)=0$, for $x \in V$, make $(I A, D)$ into a cylinder on $(A, d)$.

Proof. To see that $p$ is a quism, define a $\left(j_{1} p, 1\right)$-derivation $J$ on $(I A, D)$ by $J(x)=0, J\left(x^{\prime}\right)=s x, J(s x)=0$, and observe that $J$ is a derivation homotopy.

Proposition 2.5. Let $f, g:(A, d) \rightarrow(B, d)$ with $A$ free. The following are equivalent:

(i) $f \simeq g$;

(ii) $f \amalg g:(A, d) \amalg(A, d) \rightarrow(B, e)$ extends over $I(A, d)$;

(iii) there is a derivation homotopy from $f$ to $g$.

This characterization of DGA homotopy, as the existence of a derivation homotopy, is a powerful one, and we shall rely upon it constantly. Using it, two 
important corollaries arise at once from an alternate way of viewing the BauesLemaire cylinder. We have described $I(T V, d)$ as generated by $\left\{x, x^{\prime}, s x\right\}$, where $x$ runs through an $R$-basis for $V$, but an equally valid generating set is $\{x, s x, D s x\}$. This is summarized by the following lemma.

Lemma 2.6. (a) $I(T V, d)=(T V, d) \amalg(T(s V \oplus D s V), D)$. Likewise, $I(T(V \oplus W), d)=(T(V \oplus W), d) \amalg(T(s V \oplus D s V), D) \amalg(T(s W \oplus D s W), D)$.

(b) Given any $f:(T V, d) \rightarrow(B, e)$ and any $R$-linear $G: V \rightarrow B$ of degree +1 , there is a unique $h: I(T V, d) \rightarrow(B, e)$ satisfying $h j_{1}=f$ and $h(s x)=$ $G(x)$ for $x \in V$.

A split inclusion $i:(A, d) \rightarrow(B, e)$ is a DGA homomorphism which has a retraction as algebras. When $A$ and $B$ are free, this means that $A=T V$, $B=T(V \oplus W)$, and $e$ extends $d$. A split inclusion of free algebras is a cofibration in the following sense.

Proposition 2.7 (Homotopy extension property). Let $i:(T V, d) \rightarrow$ $\left(T(V \oplus W), d^{\prime}\right)$ be a split inclusion of free dga's. Let $f:\left(T(V \oplus W), d^{\prime}\right) \rightarrow$ $(B, e)$, and let $F: I T V \rightarrow B$ be a homotopy between $\left.f\right|_{T V}$ and some other homomorphism $g$. Then there is an extension $\widetilde{F}$ of $F$ to a homotopy between $f$ and an extension $\tilde{g}$ of $g$.

Proof. Using Lemma 2.6(a), retract $I T(V \oplus W)$ onto $T(V \oplus W) \amalg_{T V} I T V$ and compose with $f \amalg F$.

In DGA, surjections are fibrations relative to free objects, in the following sense.

Proposition 2.8 (Homotopy lifting property). Let $p:(C, \gamma) \rightarrow(B, e)$ be surjective. Let $f:(A, d) \rightarrow(C, \gamma)$ with $A=T V$, and let $F: I A \rightarrow B$ be a homotopy between $p f$ and some other homomorphism $g$. Then there exists a homotopy $\widetilde{F}$ between $f$ and a homomorphism $\tilde{g}$ such that $p \widetilde{F}=F$ and $p \tilde{g} \simeq g$.

Proof. Let $F: I A \rightarrow B$ be the homotopy. Choose any $R$-linear lifting $G$ of $\left.F\right|_{s V}$ and apply Lemma $2.6(\mathrm{~b})$.

Definition. In any category with a concept of homotopy satisfying Lemma 2.2, we call a morphism $f: A \rightarrow B$ a homotopy equivalence if there exists $g: B \rightarrow A$ for which $g f \simeq 1_{A}$ and $f g \simeq 1_{B}$. The morphism $g$ is called a homotopy inverse for $f$. Two objects have the same homotopy type if there is a homotopy equivalence between them.

In DGA we have a particularly elegant characterization of homotopy equivalences between free objects: they are precisely the quasi-isomorphisms. While it is trivial that a homotopy equivalence is a quism, the converse depends upon the following. 
Proposition 2.9 (Lifting lemma). Let $p:(C, \gamma) \rightarrow(B, e)$ be a quasi-isomorphism. Let $i:(T V, d) \rightarrow(T(V \oplus W), d)$ be a split inclusion of free dga's. Let $f:(T(V \oplus W), d) \rightarrow(B, e)$ and put $f^{\prime}=\left.f\right|_{T V}$.

(a) Suppose $p$ is surjective. Given any lifting $\tilde{f}^{\prime}:(T V, d) \rightarrow(C, \gamma)$ of $f^{\prime}$, i.e., $p \tilde{f}^{\prime}=f^{\prime}$, we may extend $\tilde{f}^{\prime}$ to a lifting $\tilde{f}$ of $f$.

(b) Suppose $p$ is surjective. Let $\tilde{f}, \tilde{g}$ be two liftings of $f$, and let $\tilde{f}^{\prime}, \tilde{g}^{\prime}$ be their restrictions to $T V$. Any homotopy $\widetilde{F}^{\prime}: I T V \rightarrow C$ between $\tilde{f}^{\prime}$ and $\tilde{g}^{\prime}$ having $p \widetilde{F}^{\prime}(s V)=0$ may be extended to a homotopy $\widetilde{F}: I T(V \oplus W) \rightarrow C$ between $\tilde{f}$ and $\tilde{g}$ having $p \widetilde{F}(s V \oplus s W)=0$.

(c) Let $\tilde{f}^{\prime}$ be any lifting of $f^{\prime}$. There is an extension $\tilde{f}$ of $\tilde{f}^{\prime}$ such ihat $p \tilde{f} \simeq f$.

(d) If $p \tilde{f} \simeq p \tilde{g}$, then $\tilde{f} \simeq \tilde{g}$.

Proof. For (a), note that $\operatorname{ker}(p)$ is an acyclic ideal, and induct on the dimension; part (b) is a special case. For (c), turn $p$ into a surjection $p^{\prime}$ via Lemma 2.1(a) and note that the left inverse for the quism $h$ of that lemma is a homotopy inverse. Now utilize the homotopy lifting property for $p^{\prime}$. Part (d) specializes (c).

Lemma 2.10. Let $(A, d)$ be free. A quism $p:(C, \gamma) \rightarrow(B, e)$ induces a bijection on sets of homotopy classes,

$$
p_{\#}:[A ; C] \stackrel{\approx}{\rightarrow}[A ; B] .
$$

Proposition 2.11. Let $(A, d)$ and $(B, e)$ be free. A homomorphism $f:(A, d) \rightarrow$ $(B, e)$ is a homotopy equivalence if and only if it is a quasi-isomorphism.

Proof. By Lemma 2.10, there exists $g: B \rightarrow A$ such that $f g \simeq 1_{B}$. Again by Lemma 2.10, there exists $f^{\prime}: A \rightarrow B$ such that $g f^{\prime} \simeq 1_{A}$. Then $f \simeq f g f^{\prime} \simeq$ $f^{\prime}$, so $g f \simeq 1_{A}$.

\section{Differential graded Lie Algebras}

We assemble here the facts we shall need involving differential graded Lie algebras. These facts fall into three subsections. First, we briefly review homotopy theory for the category $\operatorname{DGL}(R)$, again omitting most proofs. We pay particular attention to the additional hypotheses needed when $R \nsupseteq \mathbf{Q}$. Second, we examine a limited situation where the concept of derivation homotopy can be invoked for Lie algebras. We digress to cover a fairly specialized application. Third, we consider in detail a certain exact sequence of $\operatorname{Der}(L)$-modules. This sequence and its splittings will provide the key ingredients for the proof of Theorem 4.8 .

References for the homotopy theory include [7, 13, 15].

Definitions. Let $R$ denote a commutative ring with unity. A graded Lie algebra over $R$ is a graded $R$-module $L=\bigoplus_{n=-\infty}^{\infty} L_{n}$, together with a bilinear pairing 
[ , ]: $L_{m} \otimes L_{n} \rightarrow L_{m+n}$ satisfying the Jacobi identities:

$$
\begin{gathered}
{[y, x]=-(-1)^{|x||y|}[x, y] ;} \\
{[x,[y, z]]=[[x, y] z]+(-1)^{|x||y|}[y,[x, z]] .}
\end{gathered}
$$

If $L_{n}=0$ for $n<t$, we call $L$ t-reduced; "1-reduced" is also called connected. An $R$-linear $F: L \rightarrow L$ of degree $r$ is called a derivation if

$$
F([x, y])=[F(x), y]+(-1)^{r|x|}[x, F(y)] .
$$

The derivations of $L$ themselves form a graded Lie algebra denoted $\operatorname{Der}(L)$.

A differential graded Lie algebra (henceforth $d g L$ ) over $R$ is a connected Lie algebra $L$ together with a derivation $\delta$ of degree -1 whose square is zero. Our notation is $(L, \delta)$ or simply $L$. The category of dgL's over $R$ is denoted $\operatorname{DGL}(R)$ or simply DGL.

In our work with dgL's the universal enveloping algebra functor $U$ from $\operatorname{DGL}(R)$ to $\operatorname{DGA}(R)$ plays a central role. Push-outs exist in DGL, and they commute with $U$. A dgL $(L, \delta)$ is free if $L$ is free on some set $S$ as a graded Lie algebra. A free dgL need not be a free object in DGL, but $(L, \delta)$ is free if and only if $U(L, \delta)$ is free. If $L$ is free on the graded set $S$ (resp. on the $R$ free module $V$ ), we write $L=\mathrm{L}_{R}\langle S\rangle$ (resp. $L=\mathscr{L} V$ ). Thus $U \mathrm{~L}_{T}\langle S\rangle=R\langle S\rangle$ and $U \mathscr{L} V=T V$.

A DGL homomorphism $f:(L, \delta) \rightarrow(M, e)$ is called a quasi-isomorphism, or quism, if and only if $U f$ is a quism in DGA. When $R \supseteq \mathbf{Q}$, this is equivalent to the criterion that $f$ induce an isomorphism on (Lie algebra) homology. When $R \nsupseteq \mathbf{Q}$, however, the two concepts generally differ. For instance, let $L=\mathrm{L}_{R}\langle x, y\rangle$ with $|x|=1,|y|=2$, and $\delta(y)=x, \delta(x)=0$. By [8], if $p^{-1} \notin R$ for some prime $p$, then $\mathbf{Z}_{p} \subseteq H_{2 p-2}(L, \delta)$. Thus the homomorphism $f: 0 \rightarrow(L, \delta)$ does not induce an isomorphism on homology, yet it is a quism because $U(0)=R$ and $U\left(\mathrm{~L}_{R}\langle x, y\rangle, \delta\right)$ are both acyclic.

Let us imitate for DGL as much as we can of the outline provided in $\S 2$ for DGA. Lemmas 2.1 and 2.2 and the definition of cylinder may be copied essentially verbatim. However, there is no analog for derivation homotopy. The canonical cylinder, due to Tanré [15], is obtained through an entirely new method.

The Tanré cylinder, defined for free dgL's in $\operatorname{DGL}(\mathbf{Q})$, is constructed as follows. Given $(\mathscr{L} V, \delta)$, put $I(\mathscr{L} V, \delta)=(I \mathscr{L} V, D)$, where $I \mathscr{L} V=$ $\mathscr{L}(V \oplus s V \oplus D s V)$ and $\left.D\right|_{\mathscr{L} V}=\delta$. The obvious inclusion quism $j_{1}: \mathscr{L} V \rightarrow$ $I \mathscr{L} V$ is one "end" of the cylinder, and $p: I \mathscr{L} V \rightarrow \mathscr{L} V$ is given on generators by $p(x)=x, p(s x)=0, p(D s x)=0$.

The tricky part is to define the other "end" of the cylinder, the inclusion $j_{2}$. Given a pointwise nilpotent derivation $\theta$ of degree zero on a graded Lie algebra $L$, observe that

$$
e^{\theta}=1_{L}+\sum_{n=1}^{\infty} \frac{\theta^{n}}{n !}
$$


defines a Lie algebra automorphism on $L$. Define a derivation $S$ of degree +1 on $I \mathscr{L} V$ by defining it on generators as $S(x)=s x, S(s x)=0, S(D s x)=0$. Then $\alpha=[D, S]=D S+S D$ is a derivation on $I \mathscr{L} V$ of degree zero. Put

$$
j_{2}=e^{\alpha} j_{1} \text {. }
$$

One sees easily that $j_{1} \amalg j_{2}:(\mathscr{L} V, \delta) \amalg(\mathscr{L} V, \delta) \rightarrow(I \mathscr{L} V, D)$ injects and that $I \mathscr{L} V \approx \mathscr{L} V \amalg \mathscr{L} V \amalg \mathscr{L}(s V)$ as Lie algebras.

This definition can break down in $\operatorname{DGL}(R) \quad(R \nsupseteq \mathbf{Q})$ if the denominators required by (8) are unavailable in $R$. Indeed, let $\rho(R)$ denote the least positive integer (if none exists put $\rho(R)=\infty$ ) which is not a unit in $R$. (9) requires that $\alpha$ be nilpotent of order at most $\rho(R)$ on $V$. We can guarantee this only by placing constraints on the range of dimensions in which $\mathscr{L} \mathrm{V}$ is generated.

Viewing $I \mathscr{L} V$ as $(\mathscr{L} V) \amalg \mathscr{L}(s V \oplus D s V)$, put a "new grading" on it by letting $\mathscr{L} V$ keep its usual grading but assigning $s V$ and $D s V$ to lie in new grade zero. Let $F^{j}$ be spanned by all terms of new grade $j$ or less. We have $D F^{j} \subseteq F^{j}$. If $\mathscr{L} V$ is $r$-reduced, then $S F^{j} \subseteq F^{j-r}$. Thus $\alpha^{\rho(R)} F^{j} \subseteq F^{-1}=0$ as long as $j<r \rho(R)$.

Definition. A free dgL or dga over $R$ is $r$-mild if it is generated as a Lie or associative algebra by the range of dimensions $r$ through $r \rho(R)-1$, inclusive. Denote by $\operatorname{DGL}_{r}(R)$ (resp. $\operatorname{DGA}_{r}(R)$ ) the full subcategory of $\mathrm{DGL}(R)$ (resp. $\operatorname{DGA}(R))$ consisting of all $r$-mild objects.

The above remarks show

Lemma 3.1. The Tanré cylinder construction yields a valid cylinder on any r-mild $d g L$.

\section{Analogous to Proposition 2.5 is}

Lemma 3.2. Let $f, g:(L, \delta) \rightarrow(M, e)$ be two homomorphisms in $\operatorname{DGL}_{r}(R)$. Then $f \simeq g$ if and only if $f \amalg g$ factors (in DGL) through the inclusion $j_{1} \amalg j_{2}$ of $L$ into its Tanré cylinder.

Although we shall not need this, Lemma 2.6 through Proposition 2.11 now have perfect analogs in $\mathrm{DGL}_{r}(R)$. In particular, since $\mathrm{DGL}_{r}(R)$ contains only free dgL's, the homotopy equivalences are precisely the quasi-isomorphisms.

Lemma 3.1 also implies that the Lemaire-Aubry theorem is valid in $\operatorname{DGL}_{r}(R)$.

Proposition 3.3 (Lemaire-Aubry theorem). Let $f, g:(L, \delta) \rightarrow(M, e)$ be homomorphisms in $\operatorname{DGL}_{r}(R)$. Then $f \simeq g$ if and only if $U f \simeq U g$ in $\operatorname{DGA}(R)$.

Proof. See [4].

As mentioned, the concept of derivation homotopy breaks down in DGL. We would like to be able to replace the multiplications in (3) by Lie brackets, but an $F$ satisfying the resulting expression cannot be well defined. We can salvage it only at the dimension where the two homomorphisms $f$ and $g$ first differ. 
The next lemma is a variation on an idea used by Lemaire and Aubry (in [4], see Lemma 3.2 and the proof of Theorem 4.1).

Notation. For a graded $R$-module $M, M_{\leq k}$ denotes $\bigoplus_{j=0}^{k} M_{j}$. For instance, $\mathscr{L}\left(V_{\leq k}\right)$ is the Lie subalgebra of $\mathscr{L} V$ generated in dimensions $k$ and smaller. Similarly, for $M_{<k}$.

Lemma 3.4. Let $V$ be an $R$-free positively graded $R$-module, and assume $V=$ $V_{\leq n}$. Let $f, g: U(\mathscr{L} V, \delta) \rightarrow(B, e)$ be two DGA homomorphisms which coincide on $\mathscr{L}\left(V_{<n}\right)$. Then a degree $+1 \quad R$-linear

$$
F:(\mathscr{L} V)_{\leq n} \rightarrow B
$$

extends to a derivation homotopy from $f$ to $g$ if and only if

$$
\begin{gathered}
F([x, y])=[F(x), f(y)]+(-1)^{|x|}[f(x), F(y)] \\
\qquad \text { for } x, y \in \mathscr{L}\left(V_{<n}\right),|x|+|y| \leq n ; \text { and } \\
\\
e F+F \delta=f-g \text { on } V .
\end{gathered}
$$

In particular, $f \simeq g$ if and only if there exists such an $F$.

Proof. Let $\widetilde{F}$ be the unique $(f, g)$-derivation extending $\left.F\right|_{V}$. Then $\widetilde{F}$ satisfies (10a), so $F$ satisfies $(10 a)$ if and only if $F$ coincides with $\widetilde{F}$ on $\left(\mathscr{L}\left(V_{<n}\right)\right)_{\leq n}$. Consequently, if $F$ satisfies (10a) and (10b), then $\widetilde{F}$ satisfies (10b) on $V$. By Lemma $2.3, \widetilde{F}$ is a derivation homotopy from $f$ to $g$ extending $F$. The converse is straightforward.

We will be interested in applying Lemma 3.4 in a setting where the target dga is a tensor product.

Notation. The subalgebra of the dga $(A, d)$ (resp. the $\operatorname{dgL}(L, \delta))$ generated by $A_{\leq n}\left(\right.$ resp. $\left.L_{\leq n}\right)$ is denoted $\left(A_{(n)}, d\right)$ (resp. $\left.\left(L_{(n)}, \delta\right)\right)$.

Lemma 3.5. Let $(\mathscr{L} V, \delta)$ be a free $d g L, V=V_{\leq n}$, and let $(B, e)$ be any dga. Let $f, g: U(\mathscr{L} V, \delta) \rightarrow(B, e) \otimes(B, e)$ be two homotopic DGA homomorphisms which coincide on $\mathscr{L}\left(V_{<n}\right)$. Suppose that $f\left(\mathscr{L}\left(V_{<n}\right)\right) \subseteq B \otimes 1+1 \otimes B$ and that $f(x)-g(x) \in B_{+} \otimes B_{+}$for $x$ belonging to $V_{n}$. Then there exists an $F$ as in Lemma 3.4 satisfying (10a) and (10b) such that $\operatorname{im}(F) \subseteq B_{+} \otimes B_{+} \cdot A$ similar result holds if $(B, e) \otimes(B, e), B_{+} \otimes B_{+}$, and $B \otimes 1+1 \otimes B$ are replaced respectively by $(B, e)^{\otimes 3}, B_{+}^{\otimes 3}$, and $B \otimes 1 \otimes 1+1 \otimes B \otimes 1+1 \otimes 1 \otimes B$.

Proof. Let $q: B \rightarrow B$ be the $R$-linear homomorphism given by $q(1)=0$ and $q(b)=b$ for $b \in B_{+}$. Let $G:(\mathscr{L} V)_{\leq n} \rightarrow B \otimes B$ be the restriction of a derivation homotopy as in Lemma 3.4, and put $F=(q \otimes q) G:(\mathscr{L} V)_{\leq n} \rightarrow$ $B_{+} \otimes B_{+}$. Since $q$ is a chain map,

$$
e^{\prime} F+F \delta=(q \otimes q)(f-g)=f-g \quad \text { on } V,
$$

where $e^{\prime}$ is the differential on $(B, e) \otimes(B, e)$. For any $a, b \in B$ and $w \in B \otimes B$ we have

$$
(q \otimes q)([w, a \otimes 1+1 \otimes b])=[(q \otimes q)(w), a \otimes 1+1 \otimes b] .
$$


Since $f(x)$ and $f(y)$ have the form $a \otimes 1+1 \otimes b$ for $x, y \in \mathscr{L}\left(V_{<n}\right)$, we obtain from (10a) (for $G$ )

$$
\begin{aligned}
F([x, y]) & =(q \otimes q) G([x, y]) \\
& =(q \otimes q)\left([G(x), f(y)]+(-1)^{|x|}[f(x), G(y)]\right) \\
& =[F(x), f(y)]+(-1)^{|x|}[f(x), F(y)]
\end{aligned}
$$

when $x, y \in \mathscr{L}\left(V_{<n}\right),|x|+|y| \leq n$. Thus $F$ has the desired properties.

For the remainder of this section, we explore the properties of $U L$ and tensor powers of $U L$ as $\operatorname{Der}(L)$-modules. Now $L$ denotes any connected graded Lie algebra which is free as an $R$-module, and $A=U L$. Write $p$ for $\rho(R)$, so $k^{-1} \in R$ for $1 \leq k<p$. We begin with a discussion about $\operatorname{Der}(L)$-modules.

A $\operatorname{Der}(L)$-module is assumed to have a graded $R$-module structure compatible with the graded $\operatorname{Der}(L)$-action. Given two $\operatorname{Der}(L)$-modules $M$ and $N$, their tensor product $M \otimes N$ becomes a $\operatorname{Der}(L)$-module by setting

$$
\delta(a \otimes b)=\delta(a) \otimes b+(-1)^{|\delta||a|} a \otimes \delta(b)
$$

for $\delta \in \operatorname{Der}(L)$. If $f: M \rightarrow M^{\prime}$ and $g: N \rightarrow N^{\prime}$ are homomorphisms of $\operatorname{Der}(L)$-modules, then so is $f \otimes g: M \otimes N \rightarrow M^{\prime} \otimes N^{\prime}$. The switching homomorphism $\tau: M \otimes N \rightarrow N \otimes M$ given by $\tau(a \otimes b)=(-1)^{|a||b|} b \otimes a$ is another $\operatorname{Der}(L)$-homomorphism. Trivially $L$, hence $L^{\otimes n}$ and hence $T L$, are $\operatorname{Der}(L)$-modules.

Using these facts, we deduce quickly that the multiplicative homomorphism $\Delta_{T L}: T L \rightarrow T L \otimes T L$, defined by $\Delta_{T L}(x)=x \otimes 1+1 \otimes x$ for $x \in L$, is a homomorphism of $\operatorname{Der}(L)$-modules. Viewing $A=U L$ as a quotient of $T L$, we see that it inherits both a $\operatorname{Der}(L)$-action and a multiplicative $\operatorname{Der}(L)$ homomorphism

$$
\Delta=\Delta_{L}: A \rightarrow A \otimes A
$$

This $\Delta$ is of course the standard coproduct on $U L$ for which each $x \in L$ is primitive. We mention once and for all that $\Delta$ makes $U L$ into a cocommutative coassociative Hopf algebra [10]. The reduced coproduct $\bar{\Delta}: A_{+} \rightarrow A_{+} \otimes A_{+}$, given by $\bar{\Delta}(x)=\Delta(x)-1 \otimes x-x \otimes 1$, is also a $\operatorname{Der}(L)$-homomorphism.

The Poincaré-Birkhoff-Witt theorem tells us that $U L$ is a filtered $\operatorname{Der}(L)$ module, the $k$ th filtration quotient being isomorphic to the $k$-fold symmetric product of $L$ with itself [13]. We shall see shortly that for $k<p$ these quotients are $\operatorname{Der}(L)$-summands of $U L$.

Notation. Let $S_{k}$ denote the symmetric group on $k$ letters. Let $\sigma \in S_{k}$ and let $\underline{x}=\left(x_{1}, \ldots, x_{k}\right)$ be a list of homogeneous elements in $L$. Define the graded $\operatorname{sign}[\sigma: \underline{x}]$ of $\sigma$ relative to $\underline{x}$ to be the sign (i.e., +1 or -1 ) that $\sigma$ has when viewed as permuting $k$ blocks of sizes $\left|x_{1}\right|, \ldots,\left|x_{k}\right|$. Put

$$
C(\underline{x})=C\left(x_{1}, \ldots, x_{k}\right)=\frac{1}{k !} \sum_{\sigma \in S_{k}}[\sigma: \underline{x}]\left(x_{\sigma(1)} \cdots x_{\sigma(k)}\right),
$$


which defines an element of $U L$ whenever $k<p=\rho(R)$. Put $A_{+}^{[1]}=L$ and for $2 \leq k<p$,

$$
A_{+}^{[k]}=\operatorname{Span}_{R}\left\{C\left(x_{1}, \ldots, x_{k}\right) \mid x_{i} \in L\right\} \subseteq U L .
$$

For $1 \leq k<p$, we know that $A_{+}^{[k]}$ is a $\operatorname{Der}(\mathrm{L})$-submodule of $A$. Furthermore, these submodules account for all of $A_{+}$in dimensions below $r p$. Define two more families of $\operatorname{Der}(L)$-modules by

$$
\begin{gathered}
\left(A_{+}^{\otimes 2}\right)^{[k]}=\bigoplus_{i=1}^{k-1} A_{+}^{[i]} \otimes A_{+}^{[k-i]} \subseteq A_{+}^{\otimes 2} \\
\left(A_{+}^{\otimes 3}\right)^{[k]}=\bigoplus_{i=1}^{k-2} \bigoplus_{j=1}^{k-i-1} A_{+}^{[i]} \otimes A_{+}^{[j]} \otimes A_{+}^{[k-i-j]} \subseteq\left(A_{+}^{\otimes 3}\right) .
\end{gathered}
$$

Direct computation reveals the following.

Lemma 3.6. $\bar{\Delta}\left(A_{+}^{[1]}\right)=0$ and $\bar{\Delta}\left(A_{+}^{[k]}\right) \subseteq\left(A_{+}^{\otimes 2}\right)^{[k]}$ for $k<p$. Specifically,

$$
\bar{\Delta} C(\underline{x})=\sum\left[\underline{x}^{\prime} ; \underline{x}^{\prime \prime}\right] C\left(\underline{x}^{\prime}\right) \otimes C\left(\underline{x}^{\prime \prime}\right) .
$$

In (12) the sum runs over all $2^{k}-2$ partitions of $(1,2, \ldots, k)$ into an ordered pair of nonempty sublists. The corresponding sublists of $\underline{x}$ are denoted $\underline{x}^{\prime}$ and $\underline{x}^{\prime \prime}$. The notation $\left[\underline{x}^{\prime} ; \underline{x}^{\prime \prime}\right]$ represents $[\sigma: \underline{x}]$, where $\sigma$ rearranges $(1,2, \ldots, k)$ so as to obtain the first sublist followed immediately by the second sublist.

We present next a kind of inverse for $\bar{\Delta}$.

Definition. For $1 \leq k<p$, let $\mu:\left(A_{+}^{\otimes 2}\right)^{[k]} \rightarrow A_{+}^{[k]}$ be given by

$$
\left\{\begin{array}{l}
\mu\left(x_{1} \otimes C\left(x_{2}, \ldots, x_{k}\right)\right)=\frac{1}{k} C\left(x_{1}, \ldots, x_{k}\right) ; \\
\mu=0 \text { on } \bigoplus_{i=2}^{k-1} A_{+}^{[i]} \otimes A_{+}^{[k-i]} .
\end{array}\right.
$$

Direct computation verifies

Lemma 3.7. (a) $\mu$ is a $\operatorname{Der}(L)$-homomorphism.

(b) $\mu \bar{\Delta}=1$ on $\bigoplus_{k=2}^{p-1} A_{+}^{[k]}$. In particular, $\bar{\Delta}$ is one-to-one on these $A_{+}^{[k]}$.

(c) $\bar{\Delta} \mu \bar{\Delta}=\underline{\Delta}$ on $\bigoplus_{k=1}^{p-1} A_{+}^{[k]}$.

There are two more specific $\operatorname{Der}(L)$-homomorphisms that we shall need.

Definition. Put $E=\left(A_{+}^{\otimes 2}\right) \oplus\left(A_{+}^{\otimes 3}\right)$ and let $\phi=\left(\phi_{1}, \phi_{2}\right): A_{+}^{\otimes 2} \rightarrow E$, where $\phi_{1}=1-\tau$ and $\phi_{2}=\bar{\Delta} \otimes 1-1 \otimes \bar{\Delta}$. For $k<p$, let $E^{[k]}=\left(A_{+}^{\otimes 2}\right)^{[k]} \oplus\left(A_{+}^{\otimes 3}\right)^{[k]}$ and note that $\phi:\left(A_{+}^{\otimes 2}\right)^{[k]} \rightarrow E^{[k]}$. Define $\lambda: E^{[k]} \rightarrow\left(A_{+}^{\otimes 2}\right)^{[k]}$ as follows:

$$
\left\{\begin{array}{l}
\lambda(a \otimes b)=-\left(\frac{i}{k}\right) \tau(a \otimes b) \quad \text { on } A_{+}^{[i]} \otimes A_{+}^{[k-i]} ; \\
\lambda(a \otimes b \otimes c)=\frac{i+j}{k}(1+\tau)(\mu(a \otimes b) \otimes c) \text { on } A_{+}^{[i]} \otimes A_{+}^{[j]} \otimes A_{+}^{[k-i-j]} .
\end{array}\right.
$$


Lemma 3.8. (a) $\phi$ and $\lambda$ are $\operatorname{Der}(L)$-homomorphisms.

(b) $\phi \bar{\Delta}=0$.

(c) $O n\left(A_{+}^{\otimes 2}\right)^{[k]}, k<p, \bar{\Delta} \mu+\lambda \phi=1$.

(d) $\phi \lambda \phi=\phi$.

(e) For $2 \leq k<p$, the following sequence is exact:

$$
0 \rightarrow\left(A_{+}^{[k]}\right) \stackrel{\bar{\Delta}}{\rightarrow}\left(A_{+}^{\otimes 2}\right)^{[k]} \stackrel{\phi}{\rightarrow} E^{[k]} .
$$

Proof. For (a), $\phi$ and $\lambda$ are built out of other known Der(L)-homomorphisms. (b) expresses precisely the cocommutativity and coassociativity of the coproduct $\Delta: A \rightarrow A \otimes A$. (c) is a direct calculation involving Lemma 3.7(b) and (12), and (d) follows from (b) and (c). For (e), Lemma 3.7(b) showed exactness at $A_{+}^{[k]}$; by $(\mathrm{c})$, if $\phi(x)=0$, then $x \in \operatorname{im}(\bar{\Delta})$.

Taking the direct sum over $k$ of the sequences (13) yields at once

Lemma 3.9. Let $L$ be $r$-reduced and free as an $R$-module. The sequence of $\operatorname{Der}(L)$-homomorphisms

$$
0 \rightarrow L \rightarrow A_{+} \stackrel{\bar{\Delta}}{\rightarrow} A_{+} \otimes A_{+} \stackrel{\phi}{\rightarrow} E
$$

is exact in dimensions below $r p$.

We can extend our results by one additional dimension.

Lemma 3.10. Extend $\mu$ over the module $\left(A_{+}^{\otimes 2}\right)_{r p}$ by defining it to be zero on $\bigoplus_{i=1}^{p-1}\left(A_{+}^{[i]} \otimes A_{+}^{[p-i]}\right)_{r p}$. Then $\mu$ is a $\operatorname{Der}(L)$-homomorphism on $\left(A_{+}^{\otimes 2}\right)_{\leq r p}$ in the sense that, whenever $\delta \in \operatorname{Der}(L)$ and $x \in A_{+}^{\otimes 2}$ with $|x| \leq r p$ and $|\delta|+|x| \leq r p$, then $\mu \delta(x)=\delta \mu(x)$. Likewise, $\lambda$ may be extended to a $\operatorname{Der}(L)$-homomorphism on $\left(A_{+}^{\otimes 2} \oplus A_{+}^{\otimes 3}\right)_{\leq r p}$.

Proof. Straightforward.

Proposition 3.11. Let $B$ be a Hopf algebra whose coproduct $\psi$ is cocommutative and coassociative in dimensions $\leq m$, where $m \leq r p-1$. Suppose $B$ is r-reduced and free as an $R$-algebra. Write $B=R\langle S\rangle$. Then the natural Hopf algebra homomorphism $f: U(\operatorname{ker} \bar{\psi}) \rightarrow B$ restricts to an isomorphism $f^{\prime}: U\left((\operatorname{ker} \bar{\psi})_{(m)}\right) \rightarrow B_{(m)}$. Moreover, $(\operatorname{ker} \bar{\psi})_{(m)} \approx \mathrm{L}_{R}\left\langle S \cap B_{\leq m}\right\rangle$, so $\mu$ is defined on $\left(B_{+}^{\otimes 2}\right)_{m+1}$, and $f^{\prime}$ extends to an epimorphism (not as Hopf algebras) $f^{\prime \prime}: U\left((\operatorname{im}(1-\mu \bar{\psi}))_{(m+1)}\right) \rightarrow B_{(m+1)}$.

Proof. For all but the last assertion, it may be assumed that $B=B_{(m)}$, and that $\psi$ is cocommutative and coassociative. Let $L=\operatorname{ker} \bar{\psi}$. We will choose, for each $x \in S$, a $\psi$-primitive $x^{\prime} \in B$ such that $x-x^{\prime} \in\left(B_{+}\right)^{2}$. Then $S^{\prime}=\left\{x^{\prime} \mid x \in S\right\}$ is isomorphic as a graded set with $S$, and the inclusion of $S^{\prime}$ into $\operatorname{ker} \bar{\psi}$ induces the Lie algebra homomorphism $g: \mathrm{L}_{R}\left\langle S^{\prime}\right\rangle \rightarrow L$. Since 
each $x^{\prime}$ differs from $x$ by a decomposable, $S^{\prime}$ generates $B$ freely. Thus the composite

$$
R\left\langle S^{\prime}\right\rangle=U \mathrm{~L}_{R}\left\langle S^{\prime}\right\rangle \stackrel{U g}{\rightarrow} U(L) \stackrel{f}{\rightarrow} B
$$

is an isomorphism of Hopf algebras, where $f$ is the standard Hopf algebra homomorphism from the enveloping algebra of the primitives. Since $\Delta_{\mathrm{L}_{R}\left\langle S^{\prime}\right\rangle}=$ $\psi$, Lemma 3.9 says that $L=\operatorname{ker} \bar{\psi}=\operatorname{ker} \bar{\Delta}_{\mathrm{L}_{R}\left\langle S^{\prime}\right\rangle}=\mathrm{L}_{R}\left\langle S^{\prime}\right\rangle$ below dimension $m+1$. This does it.

Suppose inductively, for some $n \leq m$, that we have constructed $x^{\prime}$ for each $x \in S$ of dimension smaller than $n$. By the argument of the preceding paragraph we may identify $B_{(n-1)}$ with $U\left(L_{(n-1)}\right)$ as Hopf algebras. In particular, the $\bar{\Delta}$ for $L_{(n-1)}$ and $\bar{\psi}$ coincide in dimensions less than $n$. Let $x \in S$, $|x|=n$, and put $y=\bar{\psi}(x)$. The cocommutativity and coassociativity of $\psi$ tell us that

$$
(1-\tau)(y)=0 \quad \text { and } \quad(\bar{\Delta} \otimes 1-1 \otimes \bar{\Delta})(y)=(\bar{\psi} \otimes 1-1 \otimes \bar{\psi})(y)=0,
$$

i.e., $\phi(y)=0$. By Lemma 3.9, $y=\bar{\Delta}(z)$ for some $z \in\left(B_{(n-1)}\right)_{n}=\left(B_{+}^{2}\right)_{n}$. Since $\bar{\Delta}$ and $\bar{\psi}$ agree on $B_{(n-1)}, \bar{\psi}(x-z)=y-\bar{\Delta}(z)=0$, i.e., $x^{\prime}=x-z$ is $\psi$-primitive. This completes the inductive step, and the proof of all but the last assertion.

For the assertion regarding $f^{\prime \prime}$, we may assume that $B=B_{(m+1)}$ and that $\psi$ first fails to be cocommutative or coassociative in dimension $m+1$. Thus $B_{(m)}=U\left((\operatorname{ker} \bar{\psi})_{(m)}\right)$, and $\mu$ is defined on $\left(B_{+}^{\otimes 2}\right)_{\leq m+1}$ (using Lemma 3.10 if $m=r p-1)$. Replace each $(m+1)$-dimensional generator $x \in S$ by $x-\mu \bar{\psi}(x)$. Since $\operatorname{im}(\mu) \subseteq\left(B_{+}\right)^{2}$, we obtain a new generating set for $B$. Recalling that $(\operatorname{ker} \bar{\psi})_{\leq m}=(\operatorname{im}(1-\mu \bar{\psi}))_{\leq m}$, we see that $f^{\prime \prime}$ surjects.

Remark. In exchange for certain limitations on $R$, we can replace the hypothesis of $B$ being free with $B$ being free as an $R$-module, without altering the conclusion that $B \approx U(\operatorname{ker} \bar{\psi})$ below dimension $m+1$. When $R$ is a field, this conclusion is implicit in [10, Proposition 4.17] for $\operatorname{char}(R)=0$, in [10, Proposition 4.21] for $\operatorname{char}(R) \neq 0$.

Finally, suppose a Hopf algebra $A$ with coproduct $\psi$ can be given a differential $d$ such that $\psi:(A, d) \rightarrow(A, d) \otimes(A, d)$ is a DGA homomorphism. If a set of generators for $A$ belongs to $L=\operatorname{ker}(\bar{\psi})$, then their boundaries (i.e., $d$-images) also lie in $L$. If $\psi$ first fails to be cocommutative or coassociative in dimension $m+1$, and if some $(m+1)$-dimensional generators for $A$ lie in $\operatorname{im}(1-\mu \bar{\psi})_{m+1}$, then their boundaries lie in $(\operatorname{im}(1-\mu \bar{\psi}))_{m}=(\operatorname{ker} \bar{\psi})_{m}=L_{m}$. This gives us at once

Corollary 3.12. Let $(A, d)$ be a free r-reduced dga, $A=T V$. Let $\psi$ be a coproduct on $A$ which is also a DGA homomorphism. Suppose that $\psi$ is cocommutative and coassociative in dimensions $\leq m$, where $m \leq r p-1$. Then there is a free graded $R$-module $W \subseteq A$ such that $W \approx V$ as $R$-modules, $W_{\leq m} \subseteq \operatorname{ker}(\bar{\psi})$, 
$\left(A_{(m)}, d\right)=U\left(\mathscr{L}\left(W_{\leq m}\right), d\right), W_{>m+1}=V_{>m+1}$, and $d\left(W_{\leq m+1}\right) \subseteq \mathscr{L}\left(W_{\leq m}\right)$. In particular, if $(A, d)$ is $r$-mild and $\psi$ is both cocommutative and coassociative, then $(A, d)$ has the form $U(\mathscr{L} W, d)$ and $\psi=\Delta_{\mathscr{L} W}$, where $(\mathscr{L} W, d)$ is an $r$-mild dgL.

\section{HOPF ALGEBRAS UP TO HOMOTOPY}

We define the category HAH of homotopy algebras up to homotopy. We observe that the properties of DGA described in $\S 2$ by and large have analogs in HAH. We discuss homotopy categories briefly and state the paper's main algebraic theorem, Theorem 4.8.

As above, $R$ denotes a commutative ring with unity, $n^{-1} \in R$ for $n<$ $p=\rho(R)$, and $\tau(a \otimes b)=(-1)^{|a||b|} b \otimes a$. Recall that $\varepsilon: A \rightarrow R$ denotes the augmentation for a graded algebra, and put $\pi_{1}=(1 \otimes \varepsilon): A \otimes A \rightarrow A$, $\pi_{2}=(\varepsilon \otimes 1): A \otimes A \rightarrow A$.

Definition 4.1. Let $(A, d) \in \operatorname{DGA}(R)$, and let $\psi(A, d) \rightarrow(A, d) \otimes(A, d)$ be a DGA homomorphism. The triple $(A, d, \psi)$ is called a Hopf algebra up to homotopy (henceforth $\mathrm{Hah}$ ) over $R$ if there exist four homotopies in DGA:

(i) between $\pi_{1} \psi$ and 1 ,

(ii) between $\pi_{2} \psi$ and 1 ,

(iii) between $\tau \psi$ and $\psi$,

(iv) between $(\psi \otimes 1) \psi$ and $(1 \otimes \psi) \psi$.

A homomorphism of Hah's is a DGA homomorphism $f:(A, d) \rightarrow\left(A^{\prime}, d^{\prime}\right)$ such that $(f \otimes f) \psi \simeq \psi^{\prime} f$. The category of Hah's over $R$ and their homomorphisms is denoted $\mathrm{HAH}(R)$, or simply HAH.

A Hah $(A, d, \psi)$ is called free, $r$-reduced, $r$-mild, etc. if $(A, d)$ is so in DGA. A homomorphism in HAH is called a surjection, quism, or split inclusion if it is one in DGA.

Lemma 4.2. The identity $1:(A, d, \psi) \rightarrow(A, d, \chi)$ is a HAH homomorphism if and only if $\psi \simeq \chi$. In this case, it is an isomorphism.

Proof. Clear.

We define cylinder and homotopy in HAH by essentially copying the definitions for these concepts in DGA. Let $(A, d, \psi) \in \mathrm{HAH}$. Given any DGA cylinder $\left(A^{*}, d^{*}\right)$ on the underlying dga $(A, d)$, put $\psi^{*}=\left(j_{1} \otimes j_{2}\right) \psi p$. Then $j_{1}, j_{2}$, and $p$ are automatically HAH homomorphisms. We deduce at once the following two lemmas.

Lemma 4.3. Let $f:(A, d, \psi) \rightarrow(B, e, \chi)$ be an HAH homomorphism and let $g:(A, d) \rightarrow(B, e)$ be a DGA homomorphism. The following are equivalent:

(i) $f \simeq g$ in $D G A$;

(ii) $g$ is an $H A H$ homomorphism and $f \simeq g$ in $H A H$. 
Lemma 4.4. Let $f:(A, d, \psi) \rightarrow(B, e, \chi)$ be an HAH homomorphism and suppose $g:(B, e) \rightarrow(A, d)$ is a homotopy inverse in DGA. Then $g \in H A H$, and $f$ and $g$ are homotopy inverses in $H A H$.

Can we prove analogs for HAH of the facts in $\S 2$ about DGA? Perhaps surprisingly, we encounter a snag right away! Lemma 2.1(a) is trivial, but for Lemma 2.1(b) we obtain only the following two special cases, which fortunately suffice for subsequent applications.

Lemma 4.5. Let $(B, e, \chi) \in H A H$ and let $f:(T V, d) \rightarrow(B, e)$ be a quism in DGA. Then there exists $\omega$ making $f:(T V, d, \omega) \rightarrow(B, e, \chi)$ into an $H A H$ quism. In particular, for any $(B, e, \chi)$, there exists a surjective quism $f:(T V, d, \omega) \rightarrow(B, e, \chi)$ in $H A H$.

Proof. Use the lifting lemma to lift $\chi f: T V \rightarrow B \otimes B$ through $f \otimes f$, obtaining $\omega: T V \rightarrow T V \otimes T V$ such that $(f \otimes f) \omega \simeq \chi f$. It remains only to check the four axioms for $(T V, d, \omega)$ to be a Hah. This comes down to four applications of Proposition 2.9(d).

Lemma 4.6. Any HAH homomorphism $f(T V, d, \psi) \rightarrow(B, e, \chi)$ may be factored as $(T V, d, \psi) \stackrel{j}{\rightarrow}(T(V \oplus W), \delta, \omega) \stackrel{q}{\rightarrow}(B, e, \chi)$, where $q$ is a quism and $j$ is a split inclusion.

Proof. Let $A=T V$. Let $q$ and $j$ be given by Lemma 2.1(b). Choose $\omega$ according to the previous lemma. Then $(T(V \oplus W), \delta, \omega) \in \mathrm{HAH}$ and $q$ is an HAH homomorphism. Since $\omega j$ and $(j \otimes j) \psi$ are both liftings (through $q \otimes q$ ) up to homotopy of $\chi f$, they are homotopic, i.e., $j$ is an HAH homomorphism.

The reader may now check that the remaining facts listed in $\S 2$ remain valid in HAH.

Let $(L, \delta) \in \mathrm{DGL}(R)$. Then $(U L, \delta, \Delta)$ is a Hah. Indeed, we have equality, not just homotopy, for the four axioms. Likewise, $U f$ is an HAH homomorphism when $f \in \mathrm{DGL}$. Let $\mathrm{HAH}_{r}(R)$ denote the full subcategory of $\mathrm{HAH}(R)$ consisting of $r$-mild objects (i.e., free, $r$-reduced, and generated in degrees below $r \rho(R))$.

Observation 4.7. We may view $U$ as a functor from $\operatorname{DGL}(R)$ to $\mathrm{HAH}(R)$ or, for any $r \geq 1$, as a functor from $\operatorname{DGL}_{r}(R)$ to $\operatorname{HAH}_{r}(R)$.

Let us briefly discuss homotopy categories and closed model categories. For any category $\mathscr{C}$ having a notion of homotopy between morphisms satisfying Lemma 2.2 , we may construct the "classical" homotopy category $\mathscr{L} \mathscr{b}$. Objects in $\mathscr{H} b \mathscr{C}$ are objects of $C$, but morphisms in $\mathscr{C} \mathscr{C} \mathscr{C}$ are homotopy classes of morphisms in $\mathscr{C}$. Two objects of the same homotopy type in $\mathscr{C}$ become isomorphic in $\mathscr{L} b \mathscr{C}$. A functor $\mathscr{F}: \mathscr{C} \rightarrow \mathscr{D}$ such that $\mathscr{F} f \simeq \mathscr{F} g$ whenever $f \simeq g$ induces a functor $\mathscr{H}(F): \mathscr{H} \mathscr{C} \rightarrow \mathscr{H} \mathscr{O D}$. In particular, the functor $U$ of Observation 4.7 induces $\mathscr{H} \mathscr{C}$. In this paper we do not actually utilize any deeper concept of homotopy category than this classical one.

Quillen [12] gave a list of axioms for a closed model category, which $\operatorname{DGA}(R)$ and DGL $(\mathbf{Q})$ satisfy. The axioms refer to three special classes of morphisms, 
called fibrations, cofibrations, and weak equivalences. On any closed model category Quillen constructed a homotopy category by formally inverting the weak equivalences.

Is $\operatorname{HAH}(R)$ a closed model category? The author does not know. One of Quillen's axioms requires that any morphism factor as in Lemma 2.1(b). This axiom might not hold for all morphisms. One can also wonder whether $\mathrm{HAH}(R)$ satisfies the weaker set of axioms discovered by Baues for a cofibration category [5]. Again this hinges on the factorization axiom, but the answer is "yes" if we restrict to the full subcategory $\mathrm{HAH}_{*}(R)$ consisting of free Hah's. The homotopy category for $\mathrm{HAH}_{*}(R)$ in Baues' sense coincides with the classical $\mathscr{L l}_{0} \mathrm{HAH}_{*}(R)$. When $\rho(R)<\infty$, the categories $\operatorname{DGL}_{r}(R)$ and $\mathrm{HAH}_{r}(R)$ are not closed model nor cofibration categories.

A functor $\mathscr{F}: \mathscr{C} \rightarrow \mathscr{D}$ is an equivalence of categories if it induces a bijection on isomorphism classes of objects, as well as bijections $\operatorname{Mor}_{\mathscr{C}}(A, B) \rightarrow$ $\operatorname{Mor}_{\mathscr{D}}(\mathscr{F} A, \mathscr{F} B)$ for each pair of objects in $\mathscr{C}$. We are at last prepared to state our principal result.

Theorem 4.8. $\mathscr{H} o(U): \mathscr{H}_{0} \mathrm{DGL}_{r}(R) \rightarrow \mathscr{H}_{0} \mathrm{HAH}_{r}(R)$ is an equivalence. Furthermore, the functor $U$ itself induces a bijection on isomorphism classes of objects.

The proof that $U$ induces a surjection will occupy all of $\S 5$, while $\S 6$ will explore naturality and uniqueness.

\section{THE SURJECTIVITY OF $U$}

We prove here what is the heart of Theorem 4.8, namely, the surjectivity of $U$ on isomorphism classes. Our method of proof is an induction on dimension. Given a Hah $(A, d, \psi)$, we construct a sequence of diagonals $\psi \simeq \psi^{1} \simeq \psi^{2} \simeq$ ... which converge to something strictly cocommutative and coassociative.

Our first two lemmas are adaptations to $\operatorname{DGA}(R)$ of results proved by Lemaire and Aubry for $\operatorname{DGL}(\mathbf{Q})$. The proofs are essentially identical to those of [4] and we omit them.

Lemma 5.1. Let $f, g, h:(T V, d) \rightarrow(B, e)$ be DGA homomorphisms which coincide on $V_{\leq n}$. Suppose $F$ and $G$ are derivation homotopies from $f$ to $g$ and from $h$ to $g$, respectively. Then there is a derivation homotopy $H$ from $f$ to $h$ such that $H=F-G$ on $T\left(V_{\leq n}\right)$.

Lemma 5.2. Let $(A, d)$ and $(B, e)$ be two dga's, $A=T V$, and let $\left\{f^{n}:(A, d)\right.$ $\rightarrow(B, e)\}_{n \geq 0}$ be a sequence of DGA homomorphisms. Suppose there exist derivation homotopies $F^{n}$ from $f^{n}$ to $f^{n+1}$. Suppose further that for some monotonically increasing integer sequence $N(n)$ such that $N(n) \rightarrow \infty$ as $n \rightarrow$ $\infty$, we have $F^{n}\left(V_{\leq N(n)}\right)=0$. Define $f:(A, d) \rightarrow(B, e)$ by letting $f(x)=$ $f^{k}(x)$ where $N(k) \geq|x|$. Then $f$ is a well-defined DGA homomorphism, and $f \simeq f^{0}$. Also, if each $f^{n}$ is an isomorphism, then $f$ is an isomorphism. 
Consider an $r$-mild $\mathrm{Hah}(A, d, \psi), A=T V$. Let $k \leq r p-1$, where $p=\rho(R)$. Suppose that $\pi_{1} \psi=1_{A}=\pi_{2} \psi$, and also that the restrictions to $T\left(V_{\leq k}\right)$ of $\psi$ and of $\tau \psi$, and of $(\psi \otimes 1) \psi$ and of $(1 \otimes \psi) \psi$, coincide. Corollary 3.12 tells us that $\left(T\left(V_{\leq k}\right), d, \psi\right)=(U L, d, \Delta)$ for some $\operatorname{dgL}(L, d)$, where $L \approx \mathscr{L}\left(V_{\leq k}\right)$. We may, therefore, build the $\operatorname{Der}(L)$-homomorphisms $\phi$ and $\lambda$ of $\S 3$. They are defined on $\left(A_{+}^{\otimes 2}\right)_{\leq k}$ and on $E_{\leq k}=\left(A_{+}^{\otimes 2}\right)_{\leq k} \oplus\left(A_{+}^{\otimes 3}\right)_{\leq k}$, respectively. Of course, Lemma 3.8 is true for this range of dimensions.

Let $q=(1-\varepsilon): A \rightarrow A_{+}$be as in the proof of Lemma 3.5. We also allow $q$ to denote any of the homomorphisms $q^{\otimes 2}: A^{\otimes 2} \rightarrow A_{+}^{\otimes 2}, q^{\otimes 3}: A^{\otimes 3} \rightarrow A_{+}^{\otimes 3}$, or $\left(q^{\otimes 2} \oplus q^{\otimes 3}\right):\left(A^{\otimes 2} \oplus A^{\otimes 3}\right) \rightarrow\left(A_{+}^{\otimes 2} \oplus A_{+}^{\otimes 3}\right)=E$.

Definition 5.3. Let $(A, d, \psi)$ be an $r$-mild $\mathrm{Hah}, A=T V$. Call $(A, d, \psi)$ $n$-perfect if

(i) $\pi_{1} \psi=1_{A}=\pi_{2} \psi$;

(ii) $\psi=\tau \psi$ and $(\psi \otimes 1) \psi=(1 \otimes \psi) \psi$ on $V_{\leq n}$; and

(iii) there exist derivation homotopies $G_{1}$ from $\psi$ to $\tau \psi$ and $G_{2}$ from $(\psi \otimes 1) \psi$ to $(1 \otimes \psi) \psi$ such that $q\left(G_{1}, G_{2}\right)\left((\operatorname{ker} \bar{\psi})_{<n}\right) \subseteq \operatorname{im}\left(1_{E}-\phi \lambda\right) \subseteq E$ (cf. the paragraph after Lemma 5.2).

The initial step of our induction is provided by

Lemma 5.4. Let $(A, d, \psi)$ be an r-mild Hah. Then there exists $\chi \simeq \psi$ such that $(A, d, \chi)$ is 1-perfect.

Proof. Let $\pi$ be the surjection $\pi=\left(\pi_{1}, \pi_{2}\right): A \otimes A \rightarrow A \times A$. Because $(A, d, \psi)$ is a Hah, there is a homotopy $\pi \psi \simeq(1,1): A \rightarrow A \times A$. Apply Proposition 2.8 to obtain a lifting $\chi$ of $(1,1)$ homotopic to $\psi$. Now (i) is satisfied. For $n=1$, (ii) follows immediately and (iii) is vacuous.

Proposition 5.5. Let $(A, d, \psi)$ be $r$-mild and n-perfect, $n<r p-1, A=T V$. Then there exists $\chi \simeq \psi$ such that $\chi$ coincides with $\psi$ on $T\left(V_{\leq n}\right)$ and $(A, d, \chi)$ is $(n+1)$-perfect. Furthermore, the derivation homotopy $F$ from $\psi$ to $\chi$ may be chosen so that $F\left(V_{i}\right)=0$ for $i<n$ and for $i>n+1$.

Proof. Without loss of generality we may replace $V$ by the $R$-module $W$ of Corollary 3.12. Then $V_{\leq n} \subseteq \operatorname{ker}(\bar{\psi})$ and $d\left(V_{\leq n+1}\right) \subseteq \mathscr{L} V_{\leq n}$. Also, since $d \in \operatorname{Der}(L)$, it acts on the $\operatorname{Der}(L)$-modules $\left(T\left(V_{\leq n}\right)\right)^{\otimes 2}$ and $\left(T\left(V_{\leq n}\right)\right)^{\otimes 3}$. Fortunately, the action of $d$ in this sense coincides with the differentials on these objects viewed as dga's. The notation " $d$ " is thus unambiguous.

Let $G_{1}, G_{2}$ be as in Definition 5.3(iii). By Definition 5.3(i) and (ii), the homomorphisms $\psi$ and $\tau \psi$ fulfill the hypotheses of Lemma 3.5. We deduce that $q G_{1}$ satisfies

$$
d\left(q G_{1}\right)+\left(q G_{1}\right) d=\psi-\tau \psi=\phi_{1} \bar{\psi} \text { on } V_{\leq n+1}
$$




$$
\begin{aligned}
\left(q G_{1}\right)[x, y]= & {\left[\left(q G_{1}\right)(x), \psi(y)\right]+(-1)^{|x|}\left[\psi(x),\left(q G_{1}\right)(y)\right] } \\
= & \varepsilon_{0}(x, y) \operatorname{ad}(y)\left(q G_{1}(x)\right)+(-1)^{|x|} \operatorname{ad}(x)\left(q G_{1}(y)\right) \\
& \quad \text { for } x, y \in L,|x|+|y| \leq n+1 .
\end{aligned}
$$

Here $\varepsilon_{0}(x, y)=-(-1)^{(|x|+1)|y|}$, and we view $\operatorname{ad}(z)$ for $z \in L$ as an element of $\operatorname{Der}(L)$.

Likewise, writing $\psi_{3}$ for $(\psi \otimes 1) \psi, q G_{2}$ satisfies

$$
\begin{aligned}
d\left(q G_{2}\right)+ & \left(q G_{2}\right) d=(\psi \otimes 1) \psi-(1 \otimes \psi) \psi=\phi_{2} \bar{\psi} \quad \text { on } V_{\leq n+1} ; \\
\left(q G_{2}\right)[x, y] & =\left[\left(q G_{2}\right)(x), \psi_{3}(y)\right]+(-1)^{|x|}\left[\psi_{3}(x),\left(q G_{2}\right)(y)\right] \\
& =\varepsilon_{0}(x, y) \operatorname{ad}(y)\left(q G_{2}(x)\right)+(-1)^{|x|} \operatorname{ad}(x)\left(q G_{2}(y)\right) \\
& \text { for } x, y \in L,|x|+|y| \leq n+1 .
\end{aligned}
$$

Since $\phi$ and $\lambda$ are $\operatorname{Der}(L)$-homomorphisms, they commute with $d$ and with any $\operatorname{ad}(z)$. Apply $\lambda \phi \lambda$ to the previous four equations and write $F$ for $\lambda \phi \lambda q\left(G_{1}, G_{2}\right):(\mathscr{L} V)_{\leq n+1} \rightarrow A \otimes A$. We obtain

$$
\begin{aligned}
d F+F d= & (\lambda \phi \lambda)\left(\phi_{1}, \phi_{2}\right) \bar{\psi}=\lambda \phi \lambda \phi \bar{\psi}=\lambda \phi \bar{\psi} \quad \text { on } V_{\leq n+1} ; \\
F[x, y]= & \varepsilon_{0}(x, y) \operatorname{ad}(y) F(x)+(-1)^{|x|} \operatorname{ad}(x) F(y) \\
= & {[F(x), \psi(y)]+(-1)^{|x|}[\psi(x), F(y)] } \\
& \text { for } x, y \in L,|x|+|y| \leq n+1 .
\end{aligned}
$$

Define $\chi^{\prime \prime}=\psi-\lambda \phi \bar{\psi}: V_{\leq n+1} \rightarrow A \otimes A$, and extend $\chi^{\prime \prime}$ uniquely to an algebra homomorphism $\chi^{\prime}: T\left(V_{\leq n+1}\right) \rightarrow A \otimes A$. By Definition 5.3(ii), $\phi \bar{\psi}$ vanishes on $V_{\leq n}$, hence on $T\left(V_{\leq n}\right)$. Thus $\chi^{\prime}$ coincides with $\psi$ and with $\psi-\lambda \phi \bar{\psi}$ on $T\left(V_{\leq n}\right)$. For $x \in V_{n+1}, d(x) \in T\left(V_{\leq n}\right)$, so

$$
\chi^{\prime} d(x)=(\psi-\lambda \phi \bar{\psi}) d(x)=d(\psi-\lambda \phi \bar{\psi})(x)=d \chi^{\prime}(x) .
$$

Hence $\chi^{\prime}$ is a DGA homomorphism. The right-hand side of (17a) is precisely $\psi-\chi^{\prime}$, so Lemma 3.4 applies to $F$. Using Lemma 3.4, $F$ extends to a derivation homotopy, also denoted $F$, from $\psi$ to $\chi^{\prime}$ on $T\left(V_{\leq n+1}\right)$.

Now apply the homotopy extension property (we may take $F\left(V_{i}\right)=0$ for $i>n+1)$ to obtain a further extension of $F$ which is a derivation homotopy from $\psi$ to some DGA homomorphism $\chi$ that extends $\chi^{\prime}$. Because $F(V) \subseteq$ $A_{+}^{\otimes 2}$, we have $\pi(\psi-\chi)=\pi(d F+F d) \subseteq \pi\left(A_{+}^{\otimes 2}\right)=0$, i.e., $\pi_{1} \chi=\pi_{1} \psi=1_{A}$ and $\pi_{2} \chi=\pi_{2} \psi=1_{A}$. We have $F\left(V_{>n+1}\right)=0$ by definition, whereas for $x \in V_{<n}$ Definition 5.3(iii) yields

$$
F(x) \in \operatorname{im}(\lambda \phi \lambda(1-\phi \lambda))=\operatorname{im}(\lambda \phi \lambda-\lambda(\phi \lambda \phi) \lambda)=0 .
$$

To verify Definition 5.3(ii) for $\chi$, let $\phi_{\chi}$ be the " $\phi$ " for the coproduct $\chi$, i.e., $\phi_{\chi}=(1-\tau, \bar{\chi} \otimes 1-1 \otimes \bar{\chi})$. Note that $\phi_{\chi}$ and $\phi\left(=\phi_{\psi}\right)$ coincide in dimensions $\leq n+1$. Hence on $V_{\leq n+1}$,

$$
\phi_{\chi} \bar{\chi}=\phi_{\chi}(\bar{\psi}-\lambda \phi \bar{\psi})=\phi(\bar{\psi}-\lambda \phi \bar{\psi})=(\phi-\phi \lambda \phi) \bar{\psi}=0 \text {. }
$$


Finally, let us construct the derivation homotopies $J_{1}$ and $J_{2}$ required by Definition 5.3(iii) for $(A, d, \chi)$. We have derivation homotopies $F$ from $\psi$ to $\chi, G_{1}$ from $\psi$ to $\tau \psi$, and $\tau F$ from $\tau \psi$ to $\tau \chi$, while all four DGA homomorphisms coincide on $V_{\leq n}$. By Lemma 5.1, there is a $J_{1}$ from $\chi$ to $\tau \chi$ such that

$$
J_{1}=-F+G_{1}+\tau F=G_{1}-\phi_{1} \lambda \phi \lambda q G \text { on } V_{\leq n},
$$

where $G=\left(G_{1}, G_{2}\right): T V \rightarrow\left(A^{\otimes 2}\right) \oplus\left(A^{\otimes 3}\right)$. The diagram of derivation homotopies

$(\chi \otimes 1) \chi \stackrel{(\chi \otimes 1) F}{\leftarrow}(\chi \otimes 1) \psi \stackrel{(F \otimes 1) \psi}{\leftarrow}(\psi \otimes 1) \psi \stackrel{G_{2}}{\rightarrow}(1 \otimes \psi) \psi$

$$
\stackrel{(1 \otimes F) \psi}{\rightarrow}(1 \otimes \chi) \psi \stackrel{(1 \otimes \chi) F}{\rightarrow}(1 \otimes \chi) \chi
$$

likewise yields a $J_{2}$ from $(\chi \otimes 1) \chi$ to $(1 \otimes \chi) \chi$ such that

$$
\begin{aligned}
J_{2} & =-(\chi \otimes 1-1 \otimes \chi) F-(F \otimes 1-1 \otimes F) \psi+G_{2} \\
& =G_{2}-\phi_{2} \lambda \phi \lambda q G-(\text { something in } \operatorname{ker}(q)) \text { on } V_{\leq n} .
\end{aligned}
$$

Thus

$$
\begin{aligned}
q\left(J_{1}, J_{2}\right) & =q(G-\phi \lambda \phi \lambda q G) \\
& =(1-\phi \lambda) q G \subseteq \operatorname{im}\left(1_{E}-\phi \lambda\right) \quad \text { on } V_{\leq n} .
\end{aligned}
$$

It remains only to pass from knowing this relation on $V_{\leq n}$ to knowing it on all of $(\operatorname{ker}(\bar{\chi}))_{\leq n}$, as needed for Definition 5.3(iii). Observe that if $b_{i} \in$ $\operatorname{ker}(\bar{\chi})$ and $q\left(J_{1}, \bar{J}_{2}\right) b_{i}=\left(1_{E}-\phi \lambda\right)\left(x_{i}\right)$ for $i=1,2$, with $\left|b_{1}\right|+\left|b_{2}\right| \leq n$, then $q\left(J_{1}, J_{2}\right)\left(\left[b_{1}, b_{2}\right]\right)=\left(1_{E}-\phi \lambda\right)(y)$, where

$$
y=(-1)^{\left|b_{1}\right|} \operatorname{ad}\left(b_{1}\right)\left(x_{2}\right)+\varepsilon_{0}\left(b_{1}, b_{2}\right) \operatorname{ad}\left(b_{2}\right)\left(x_{1}\right) .
$$

This completes the proof.

Theorem 5.6. The functor $U: \mathrm{DGL}_{r}(R) \rightarrow \mathrm{HAH}_{r}(R)$ surjects on isomorphism classes. I.e., for any $(A, d, \psi) \in \operatorname{HAH}_{r}(R)$, there exists $\chi \simeq \psi$ such that $(A, d, \chi)=(U L, d, \Delta)$ for some $(L, d) \in \mathrm{DGL}_{r}(R)$.

Proof. Applying Proposition 5.5 inductively, we obtain a sequence $\psi \simeq \psi^{1} \simeq$ $\psi^{2} \simeq \cdots$ for which $\left(A, d, \psi^{n}\right)$ is $n$-perfect. If $p=\rho(R)<\infty$, the sequence terminates at $\psi^{r p-1}$ but $\psi^{r p-1}$ is strictly cocommutative and coassociative. By Corollary 3.12, $\left(A, d, \psi^{r p-1}\right)=(U L, d, \Delta)$. If instead $\rho(R)=\infty$, Lemma 5.2 shows that $\psi$ is homotopic to some "limit coproduct" $\chi$ which is cocommutative and coassociative. Again apply Corollary 3.12.

\section{NATURALITY AND UNIQUENESS}

We complete the proof of Theorem 4.8. We demonstrate that any homomorphism $f$ between enveloping algebras in $\mathrm{HAH}_{r}(R)$ is homotopic to $U \alpha$ for 
some homomorphism $\alpha$ in $\operatorname{DGL}_{r}(R)$. By the Lemaire-Aubry theorem (Proposition 3.3), the homotopy class of $\alpha$ is unique. If $f$ is an isomorphism, $\alpha$ may be taken to be an isomorphism. Thus $U$ induces an injection on isomorphism classes. Combining these facts with Theorem 5.6, we easily obtain the desired equivalence of homotopy categories.

We begin with a definition, an inductive step, and a theorem, which are analogs of Definition 5.3, Proposition 5.5, and Theorem 5.6. We retain the notation $q$ for the retraction $q: B \otimes B \rightarrow B_{+} \otimes B_{+}$.

Definition 6.1. Let $(\mathscr{L} V, d)$ be an $r$-mild dgL and let $(M, e)$ be an $r$-reduced $R$-free dgL. An HAH homomorphism $f: U(\mathscr{L} V, d) \rightarrow U(M, e)$ is n-perfect if

(i) $f\left(V_{\leq n}\right) \subseteq M$; and

(ii) there is a derivation homotopy $G$ from $\Delta_{M} f$ to $(f \otimes f) \Delta_{L}$ such that $q G\left(V_{<n}\right) \subseteq \operatorname{im}\left(1-\bar{\Delta}_{M} \mu\right) \subseteq\left((U M)_{+}\right)^{\otimes 2}$, where $\mu$ is the $\operatorname{Der}(M)$ homomorphism of $\S 3$.

Remark. Any such $f$ is automatically 1-perfect.

Proposition 6.2. Let $(\mathscr{L} V, d) \in \mathrm{DGL}_{r}(R)$, let $(M, e)$ be an r-reduced $R$-free $d g L$, and let $f: U(\mathscr{L} V, d) \rightarrow U(M, e)$ be n-perfect, where $n<r p-1, p=$ $\rho(R)$. There is an $(n+1)$-perfect $g$ such that $g \simeq f$. If $f$ is an isomorphism, so is $g$. There is a derivation homotopy $F$ from $f$ to $g$ having $F\left(V_{i}\right)=0$ for $i<n$ and for $i>n+1$.

Proof. Write $L=\mathscr{L} V, B=U M$, and let $\Delta_{L}, \Delta_{M}$ denote the respective coproducts on $U L, B$. Let $\mu:\left(B_{+}^{\otimes 2}\right)_{\leq r p} \rightarrow\left(B_{+}\right)_{\leq r p}$ be the $\operatorname{Der}(M)$-homomorphism defined in $\S 3$. It satisfies $\bar{\Delta}_{M} \mu \bar{\Delta}_{M}=\bar{\Delta}_{M}$ and $\mu \bar{\Delta}_{M} \mu=\mu$ below dimension $r p$. Since $e \in \operatorname{Der}(M)$, it acts on $B \otimes B$, and this action coincides with the differential on $B \otimes B$ as a dga. Provided we stay at or below dimension $r p, \mu$ commutes with $e$ and with any $\operatorname{ad}(z)$ for $z \in M$.

Note that $\Delta_{M} f$ and $(f \otimes f) \Delta_{L}$ are two DGA homomorphisms from $(U L, d)$ to $(B \otimes B, e)$ which coincide on $V_{\leq n}$. The restriction of $(f \otimes f) \Delta_{L}$ to $\mathscr{L} V$ lies in $B \otimes 1+1 \otimes B$, and the difference

$$
\Delta_{M} f-(f \otimes f) \Delta_{L}=\bar{\Delta}_{M} f
$$

automatically takes its image in $B_{+} \otimes B_{+}$. Apply Lemma 3.5 to the $G$ given by Definition 6.1 (ii). We see that $q G$ satisfies

$$
\begin{gathered}
e(q G)+(q G) d=\bar{\Delta}_{M} f \text { on } V_{\leq n+1} \\
(q G)[x, y]=\left[(q G)(x), \Delta_{M} f(y)\right]+(-1)^{|x|}\left[\Delta_{M} f(x),(q G)(y)\right] \\
=\varepsilon_{0}(x, y) \operatorname{ad}(f(y))(q G(x))+(-1)^{|x|} \operatorname{ad}(f(x))(q G(y)) \\
\text { for } x, y \in \mathscr{L}\left(V_{\leq n}\right),|x|+|y| \leq n+1 .
\end{gathered}
$$

Here $\varepsilon_{0}(x, y)=-(-1)^{(|x|+1)|y|}$ as in $\S 5$. 
Apply $\mu$ to both of these equations and write $F=\mu q G$. We obtain

$$
\begin{gathered}
e F+F d=\mu \bar{\Delta}_{M} f \quad \text { on } V_{\leq n+1} ; \\
F[x, y]=\varepsilon_{0}(x, y) \operatorname{ad}(f(y))(F(x))+(-1)^{|x|} \operatorname{ad}(f(x))(F(y)) \\
=\left[F(x), \Delta_{M} f(y)\right]+(-1)^{|x|}\left[\Delta_{M} f(x), F(y)\right] \\
\quad \text { for } x, y \in \mathscr{L}\left(V_{\leq n}\right),|x|+|y| \leq n+1 .
\end{gathered}
$$

Put $g^{\prime \prime}=f-\mu \bar{\Delta}_{M} f: V_{\leq n+1} \rightarrow B$ and extend to an algebra homomorphism $g^{\prime}: T\left(V_{\leq n+1}\right) \rightarrow B$. Then $g^{\prime}$ coincides on $T\left(V_{\leq n}\right)$ with $f$, and $\bar{\Delta}_{M} f(d x) \in$ $\bar{\Delta}_{M}(M)=0$ for $x \in V_{\leq n+1}$. Thus

$$
g^{\prime} d(x)=\left(f-\mu \bar{\Delta}_{M} f\right) d(x)=e\left(f-\mu \bar{\Delta}_{M} f\right)(x)=e g^{\prime}(x)
$$

for $x \in V_{\leq n+1}$, so $g^{\prime}$ is a DGA homomorphism. Applying Lemma 3.4 to $f$ and $g^{\prime}$, deduce from $(19 \mathrm{a}, \mathrm{b})$ that $F$ extends to a derivation homotopy, also denoted $F$, from $f$ to $g^{\prime}$ on $T\left(V_{\leq n+1}\right)$.

Using the homotopy extension property, extend $F$ to a derivation homotopy from $f$ to some DGA homomorphism $g$ that extends $g^{\prime}$. Recall that $\operatorname{im}(\mu) \subseteq$ $\left(B_{+}\right)^{2}$. Consequently, choosing $F\left(V_{>n+1}\right)=0$, we have $\operatorname{im}(F) \subseteq\left(B_{+}\right)^{2}$. It follows that $f(x)-g(x)$ is decomposable for each $x \in V$. Thus $f$ is an isomorphism if and only if $g$ is an isomorphism.

Notice that $\bar{\Delta}_{M} g(x)=\left(\bar{\Delta}_{M}-\bar{\Delta}_{M} \mu \bar{\Delta}_{M}\right) f(x)=0$ for $x \in V_{\leq n+1}$, i.e., $g\left(V_{\leq n+1}\right)$ $\subseteq M$. Use Definition 6.1(ii) to see that

$$
F\left(V_{<n}\right)=\mu q G\left(V_{<n}\right) \subseteq \operatorname{im}\left(\mu\left(1-\bar{\Delta}_{M} \mu\right)\right)=0 .
$$

Finally, by Lemma 5.1, we may add the derivation homotopies indicated by the diagram

$$
\Delta_{M} g \stackrel{\Delta_{M} F}{\underline{L}} \Delta_{M} f \stackrel{G}{\rightarrow}(f \otimes f) \Delta_{L} \stackrel{(F \otimes f) \Delta_{L}}{\rightarrow}(g \otimes f) \Delta_{L} \stackrel{(g \otimes F) \Delta_{L}}{\rightarrow}(g \otimes g) \Delta_{L} .
$$

We obtain a derivation homotopy $J$ from $\Delta_{M} g$ to $(g \otimes g) \Delta_{L}$ satisfying

$$
\begin{aligned}
J & =-\Delta_{M} F+G+(F \otimes f+g \otimes F) \Delta_{L} \\
& =G-\Delta_{M} \mu q G+(\text { something in } \operatorname{ker}(q)) \text { on } V_{\leq n} .
\end{aligned}
$$

Hence $q J=q G-\bar{\Delta}_{M} \mu q G=\left(1-\bar{\Delta}_{M} \mu\right) q G$ on $V_{\leq n}$.

Theorem 6.3. Let $f: U(L, d) \rightarrow U(M, e)$ be a homomorphism in $\mathrm{HAH}(R)$, where $L$ is $r$-mild and $M$ is r-reduced and $R$-free. There exists a homomorphism $\alpha$ in $\operatorname{DGL}(R)$ such that $f \simeq U \alpha$. If $f$ is an isomorphism then $\alpha$ is an isomorphism.

Proof. Observe that $g=U \alpha$ for some $\alpha$ if and only if $g(L) \subseteq M$, and that $U \alpha$ is an isomorphism if and only if $\alpha$ is an isomorphism. By Proposition 6.2 we have a sequence $f=f^{1} \simeq f^{2} \simeq \cdots$, where $f^{n}$ is $n$-perfect. When $f$ is an isomorphism, so is each $f^{n}$. If $\rho(R)=p<\infty$, then $f^{r p-1}(L) \subseteq M$, and 
we are done. If $\rho(R)=\infty$, Lemma 5.2 shows that $f$ is homotopic to some $g$ having $g(L) \subseteq M$, with $g$ being an isomorphism if $f$ is.

Theorem 6.4. Let $f:(A, d, \psi) \rightarrow(B, e, \chi)$ be any homomorphism in $\mathrm{HAH}_{r}(R)$. There exists a homomorphism $\alpha:(L, d) \rightarrow(M, e)$ in $\operatorname{DGL}_{r}(R)$ such that $U(L, d)$ may be identified with $(A, d) ; U(M, e)$ may be identified with $(B, e)$; and there exist DGA homotopies $f \simeq U \alpha, \psi \simeq \Delta_{L}, \chi \simeq \Delta_{M}$. Furthermore, $\alpha$ is an isomorphism if and only if $f$ is an isomorphism.

Proof. Use Theorem 5.6 to choose $(L, d)$ and $(M, e)$. Then use Theorem 6.3 to choose $\alpha$.

Corollary 6.5 (cf. 4.8). The functor $U: \mathrm{DGL}_{r}(R) \rightarrow \mathrm{HAH}_{r}(R)$ induces a bijection on isomorphism classes.

Theorem 6.6. Let $(L, d)$ and $(M, e)$ be r-mild $d g L$ 's. Suppose there is an $H A H$ homotopy equivalence $f:\left(U L, d, \Delta_{L}\right) \rightarrow\left(U M, e, \Delta_{M}\right)$. Then $(L, d)$ and $(M, e)$ have the same DGL homotopy type.

Proof. Let $g$ be a homotopy inverse in $\mathrm{HAH}_{r}(R)$ for $f$ (cf. Lemma 4.4). By Theorem 6.3 choose $\alpha: L \rightarrow M$ and $\beta: M \rightarrow L$ such that $U \alpha \simeq f$ and $U \beta \simeq g$. Then $U(\beta \alpha) \simeq U\left(1_{L}\right)$, so the Lemaire-Aubry theorem says that $\beta \alpha \simeq 1_{L}$ in $\operatorname{DGL}_{r}(R)$. Likewise, $\alpha \beta \simeq 1_{M}$ in $\operatorname{DGL}_{r}(R)$.

Corollary 6.7. The functor $U: \operatorname{DGL}_{r}(R) \rightarrow \mathrm{HAH}_{r}(R)$ induces a bijection on homotopy types.

This corollary, together with Theorem 6.3 and the Lemaire-Aubry theorem, yield at once

Theorem 6.8 (cf. 4.8). The functor $U$ induces an equivalence

$$
\mathscr{H}_{a}(U): \mathscr{H}_{a} \mathrm{DGL}_{r}(R) \rightarrow \mathscr{H}_{a} \mathrm{HAH}_{r}(R) .
$$

\section{THE SINGULAR CHAINS ON A TOPOLOGICAL MONOID}

We apply Theorem 4.8 to the cubical singular chain complex of a topological monoid. Over a ring containing the rationals, we find a strictly cocommutative coassociative diagonal approximation. Using it, we construct a functor $E$ from topological monoids to DGL whose composite with the enveloping algebra functor equals the Eilenberg subcomplex of the (cubical) singular chain complex. We use $E$ to construct rational "commutative cochains."

We begin by introducing some formalism which will facilitate our study of the cubical singular chains on a topological monoid. Let $R$ be an arbitrary commutative ring with unity. Let $\mathrm{CU}_{*}(X)$ denote the cubical singular chain complex on an arbitrary topological space $X$ with coefficients in $R$. To construct $\mathrm{CU}_{*}(X)$, take the $R$-module generated freely by all the singular $n$-cubes (i.e., continuous $\sigma: I^{n} \rightarrow X$, where $I^{n}$ is the unit cube), and divide out by the $R$-submodule generated by degenerate cubes ( $\sigma$ is degenerate if it factors 
through any of the $n$ standard projections onto $\left.I^{n-1}\right)$. As is well known $\mathrm{CU}_{*}()$ is naturally chain equivalent with the functor $C_{*}()$ of simplicial singular chains.

Define a nonconnected graded algebra $S$ by $S=R\langle a, b, x\rangle$, where $|a|=$ $|b|=0$ and $|x|=1$. Impose a second grading on $S$ by word length, $l(a)=$ $l(b)=l(x)=1$. We interpret $x, a, b$ as symbolizing the interval $I=[0,1]$ and its endpoints 0 and 1 , respectively. Words of length $n$ in $S$ correspond bijectively to faces of the $n$-cube $I^{n}$ according to the following scheme. A word $u$ on $\{a, b, x\}$ corresponds to a face of dimension $|u|$ (= the number of $x$ 's). The $i$ th coordinate of the face inclusion map, $1 \leq i \leq n$, is constant at 0 (resp. 1 ) if the $i$ th letter of $u$ is " $a$ " (resp. " $b$ "). In this way, we view the words $u$ in $S$ of length $n$ as elements of $\mathrm{CU}_{*}\left(I^{n}\right)$.

Let $D: S \rightarrow S$ be the $(1,1)$-derivation on $S$ of degree -1 satisfying $D(a)=$ $D(b)=0$ and $D(x)=b-a$. Given a word $u \in S$ of length $n$ and a singular $n$-cube $\sigma: I^{n} \rightarrow X$, let $\sigma \circ u$ denote the restriction of $\sigma$ to the face $u$. Then the boundary of $\sigma$ is given by the formula

$$
\partial(\sigma)=\sigma \circ D\left(x^{n}\right) .
$$

Using this formalism we may also give explicit Eilenberg-Zilber homomorphisms

$$
\mathrm{CU}_{*}(X \times Y) \underset{\eta}{\rightarrow} \mathrm{CU}_{*}(X) \otimes \mathrm{CU}_{*}(Y) \underset{\kappa}{\rightarrow} \mathrm{CU}_{*}(X \times Y)
$$

such that $\eta \kappa$ is the identity and $\kappa \eta$ is naturally chain homotopic to the identity. When $\alpha: I^{i} \rightarrow X$ and $\beta: I^{j} \rightarrow Y$, we define $\kappa(\alpha \otimes \beta)$ to be the singular cube $\alpha \times \beta: I^{i+j}=I^{i} \times I^{j} \rightarrow X \times Y$.

To give the formula for $\eta$ we introduce $\Psi: S \rightarrow S \otimes S$, which is the unique algebra homomorphism satisfying

$$
\Psi(a)=a \otimes a, \quad \Psi(b)=b \otimes b, \quad \Psi(x)=a \otimes x+x \otimes b .
$$

Notice that $D \Psi=\Psi D$, where $D$ denotes either the differential we defined above or the differential it induces on $S \otimes S$. For $\left(\sigma, \sigma^{\prime}\right): I^{n} \rightarrow X \times Y$, put

$$
\eta\left(\sigma, \sigma^{\prime}\right)=\left(\sigma \otimes \sigma^{\prime}\right) \circ \Psi\left(x^{n}\right) .
$$

We leave it to the reader to verify that $\eta \kappa=1$ and $\kappa \eta \simeq 1$. Putting $\psi=$ $\eta \circ \mathrm{CU}_{*}(\Delta)$, where $\Delta: X \rightarrow X \times X$ is the diagonal map, we obtain the formula

$$
\psi(\sigma)=(\sigma \otimes \sigma) \circ \Psi\left(x^{n}\right), \quad \sigma: I^{n} \rightarrow X,
$$

for the diagonal approximation (in the Alexander-Whitney sense) $\psi$.

Let $\mathrm{MON}_{r}$ denote the category of $(r-1)$-connected topological monoids, $r \geq 0 \quad$ (MON $_{0}$ contains all topological monoids). For $X \in \mathbf{M O N}_{r}$, we use $\kappa$ to give $\mathrm{CU}_{*}(X)$ the obvious associative multiplication and unit. Clearly, $\partial$ is a derivation. For $X, Y \in \mathrm{MON}_{0}$, it is easily checked that

$$
\eta\left(\left(\sigma_{1}, \sigma_{1}^{\prime}\right) \cdot\left(\sigma_{2}, \sigma_{2}^{\prime}\right)\right)=\eta\left(\sigma_{1}, \sigma_{1}^{\prime}\right) \cdot \eta\left(\sigma_{2}, \sigma_{2}^{\prime}\right) .
$$

Hence, $\psi$ is also multiplication-preserving. 
Because $\Psi$ is strictly coassociative, so is $\psi$. We can define an explicit derivation homotopy $E^{\prime}$ from $\Psi$ to $\tau \Psi$ by setting

$$
E^{\prime}(a)=0, \quad E^{\prime}(b)=0, \quad E^{\prime}(x)=-x \otimes x .
$$

When $X \in \mathrm{MON}_{0}$, the formula

$$
E(\sigma)=(\sigma \otimes \sigma) E^{\prime}\left(x^{n}\right)
$$

gives an explicit natural derivation homotopy between $\psi$ and $\tau \psi$.

When $X$ is an $(r-1)$-connected pointed space, $r \geq 1$, let $\mathrm{CU}_{*}^{r}(X)$ denote its $(r-1)$ st Eilenberg subcomplex, i.e., the subcomplex of $\mathrm{CU}_{*}(X)$ generated by all singular cubes which send the $(r-1)$-skeleton of $I^{n}$ to the base point. When $X \in \mathrm{MON}_{r}, r \geq 1$, then $\mathrm{CU}_{*}^{r}(X)$ is an $r$-reduced dga which is quasiisomorphic to $\mathrm{CU}_{*}(X)$. We have actually proved

Proposition 7.1. Let $r \geq 1$. Then $\operatorname{CU}_{*}^{r}()$, together with the coproduct $\psi$ of (21), is a functor from $\mathrm{MON}_{r}$ to $\mathrm{HAH}(R)$. The coproduct satisfies strict coassociativity and naturality, and $\pi_{1} \psi=\pi_{2} \psi=1$. The homotopy $E$ from $\psi$ to $\tau \psi$ is given by (23).

We want to apply Theorem 4.8 to $\left(\mathrm{CU}_{*}^{r}(X), \psi\right)$, but we cannot do so directly because $\mathrm{CU}_{*}^{r}(X)$ might not be a tensor algebra. We proceed instead to define a formal object which acts as a universal example for a singular cube on a topological monoid. This formal object contains all faces of $\sigma$ and all products of faces of $\sigma$. It is a $\mathrm{Hah}$, and we apply Theorem 4.8 to it. In our discussion we incorporate the connectivity parameter $r$. Over $\mathbf{Q}$ it suffices to put $r=1$.

Fix $r \geq 1$. Let $S^{r}=S_{\geq r}=\operatorname{Span}\{$ words $u \in S=R\langle a, b, x\rangle$ which utilize $r$ or more $x$ 's $\}$. Define a graded $R$-module homomorphism $\gamma: S \rightarrow T S^{r}$ by letting $\left.\gamma\right|_{S^{r}}$ be the inclusion of $S^{r}=T^{1} S^{r}$ into $T S^{r}$ and putting $\gamma(u)=0$ if $1 \leq|u|<r$ and $\gamma(u)=1$ if $u$ is a word of dimension zero. Thus $\operatorname{ker}(\gamma)$ is spanned by all words $u$ having $0<|u|<r$ together with all differences $u-v$, where $u$ and $v$ are words of dimension zero. Note that

$$
D(\operatorname{ker}(\gamma)) \subseteq \operatorname{ker}(\gamma), \quad \Psi(\operatorname{ker}(\gamma)) \subseteq \operatorname{ker}(\gamma \otimes \gamma), \quad E^{\prime}(\operatorname{ker}(\gamma)) \subseteq \operatorname{ker}(\gamma \otimes \gamma)
$$

Also, $\operatorname{im}(\gamma)$ generates $T S^{r}$ as an algebra.

Because of (24), we may define a $(1,1)$-derivation $D^{r}: T S^{r} \rightarrow T S^{r}$ of degree -1 , an algebra homomorphism $\Psi^{r}: T S^{r} \rightarrow T S^{r} \otimes T S^{r}$, and a $\left(\Psi^{r}, \tau \Psi^{r}\right)$ derivation of degree +1 by the formulas

$$
D^{r} \gamma=\gamma D, \quad \Psi^{r} \gamma=(\gamma \otimes \gamma) \Psi, \quad E^{r} \gamma=(\gamma \otimes \gamma) E^{\prime} .
$$

Note that $\left(D^{r}\right)^{2}=0$ and (by Lemma 2.3) that $E^{r}$ is a derivation homotopy from $\Psi^{r}$ to $\tau \Psi^{r}$. Also, $\pi_{1} \Psi^{r}=\pi_{2} \Psi^{r}=1$, and $\Psi^{r}$ is coassociative because $\Psi$ 
is. We have proved

Lemma 7.2. $\left(T S^{r}, D^{r}, \Psi^{r}\right)$ is an r-reduced Hah.

We want to apply Thecrem 5.6 to the Hah $\left(T S^{r}, D^{r}, \Psi^{r}\right)$. However, we will not simply plug this Hah into the theorem, because we care that certain compatibilities should hold for the resulting coproduct. Observe first that $\left(T S^{r}, D^{r}, \Psi^{r}\right)$ actually decomposes as

$$
T S^{r}=\amalg_{n=r}^{\infty} T S^{r, n},
$$

where $S^{r, n}=\left\{u \in S_{\geq r} \mid l(u)=n\right\}$. The formulas for $D^{r}$ and $\Psi^{r}$ are compatible with this decomposition. If $u$ is any word on $\{a, b, x\}$ of length $n$ and dimension $m$, write $u=y_{0} x y_{1} x \cdots x y_{m}$, where each $y_{j}$ is a word on the set $\{a, b\}$. Then $u$ determines an HAH homomorphism $h_{u}:\left(T S^{r, m}, D^{r}, \Psi^{r}\right) \rightarrow$ $\left(T S^{r, n}, D^{r}, \Psi^{r}\right)$ by the formula

$$
h_{u}\left(\gamma\left(z_{1} \cdots z_{m}\right)\right)=\gamma\left(y_{0} z_{1} y_{1} z_{2} \cdots z_{m} y_{m}\right),
$$

$z_{j} \in\{a, b, x\}$. In particular, $h_{u}\left(\gamma\left(x^{m}\right)\right)=\gamma(u)$.

Now we carefully construct the derivation homotopy $F^{r}$ from $\Psi^{r}$ to the new coproduct $\Phi^{r}$, so that $F^{r}$ and $\Phi^{r}$ commute with every $h_{u}$ (this means: $\left.F^{r} h_{u}=\left(h_{u} \otimes h_{u}\right) F^{r}, \Phi^{r} h_{u}=\left(h_{u} \otimes h_{u}\right) \Phi^{r}\right)$. Suppose for some $n$ that $\Phi^{r}$ and $F^{r}$ have been defined on $T S^{r}, k$ for $k \leq n$ and that they commute with $h_{u}$ whenever $l(u)<n$. Suppose further that $\left(T S^{r, k}, D^{r}, \Phi^{r}\right)$ is $k$-perfect in such a way that the $G_{1}$ and $G_{2}$ of Definition 5.3 (iii) may be chosen so as to be compatible with all such $h_{u}$ 's. When $u$ is a word of length $n$ and dimension $m<n$, put

$$
\Phi^{r} \gamma(u)=\left(h_{u} \otimes h_{u}\right) \Phi^{r} \gamma\left(x^{m}\right), \quad F^{r} \gamma(u)=\left(h_{u} \otimes h_{u}\right) F^{r} \gamma\left(x^{m}\right) .
$$

This suffices to define $\Phi^{r}$ and $F^{r}$ on the sub-dga $\left(T S^{r, n}\right)_{(n-1)}$.

Thus $F^{r}$ is a derivation homotopy from the $\left(T S^{r, n}\right)_{(n-1)}$ restriction of $\Psi^{r}$ to $\Phi^{r}$. It remains only to extend $\Phi^{r}$ and $F^{r}$ over the single $n$-dimensional generator $\gamma\left(x^{n}\right)$ of $T S^{r, n}$. Proposition 5.5 will do this provided that $n<$ $r \rho(R)$ and provided that $\left(\left(T S^{r, n}\right)_{(n-1)}, D^{r}, \Phi^{r}\right)$ is $(n-1)$-perfect. The latter requirement is met via our inductive hypothesis that $G_{1}$ and $G_{2}$ commute with each $h_{u}$. We have outlined a proof for

Lemma 7.3. If $\mathbf{Q} \subseteq R$, there is a derivation homotopy $F^{r}$ from $\Psi^{r}$ to a cocommutative coassociative coproduct $\Phi^{r}$ on $\left(T S^{r}, D^{r}\right)$ such that $F^{r}$ and $\Phi^{r}$ commute with any $h_{u}$. If $p=\rho(R)<\infty$, then $F^{r}$ and $\Phi^{r}$ exist commuting with $h_{u}$ 's on $T S^{r, n}$ for $n<r p$.

Now let $X \in \mathrm{MON}_{r}$ and let $w \in T S^{r, n}$, where $n<r p$. Let $\sigma: I^{n} \rightarrow X$ send the $(r-1)$-skeleton of $I^{n}$ to the base point. If $w=\gamma\left(u_{1}\right) \gamma\left(u_{2}\right) \cdots \gamma\left(u_{m}\right)$, $u_{j} \in S, l\left(u_{j}\right)=n$, then $\sigma \square w$ denotes the product

$$
\sigma \square w=\left(\sigma \circ u_{1}\right) \cdot\left(\sigma \circ u_{2}\right) \cdots\left(\sigma \circ u_{m}\right)
$$


in the dga $\mathrm{CU}_{*}^{r}(X)$. Notice that $\sigma \square \gamma(u)=\sigma \circ u$ for $u \in S$, so

$$
\begin{aligned}
(\sigma \otimes \sigma) \square \Psi^{r} \gamma\left(x^{n}\right) & =(\sigma \otimes \sigma)(\gamma \otimes \gamma) \Psi\left(x^{n}\right) \\
& =(\sigma \otimes \sigma) \circ \Psi\left(x^{n}\right)=\psi(\sigma) .
\end{aligned}
$$

Similarly, $\partial(\sigma \square w)=\sigma \square\left(D^{r} w\right)$.

Given $X, Y \in \mathrm{MON}_{r}$, define $F^{\prime}, \eta^{\prime}: \mathrm{CU}_{*}^{r}(X \times Y) \rightarrow \mathrm{CU}_{*}^{r}(X) \otimes \mathrm{CU}_{*}^{r}(Y)$ by

$$
\begin{aligned}
& \eta^{\prime}\left(\sigma, \sigma^{\prime}\right)=\left(\sigma \otimes \sigma^{\prime}\right) \oslash \Phi^{r} \gamma\left(x^{n}\right), \\
& F^{\prime}\left(\sigma, \sigma^{\prime}\right)=\left(\sigma \otimes \sigma^{\prime}\right) \oslash F^{r} \gamma\left(x^{n}\right),
\end{aligned}
$$

where $|\sigma|=\left|\sigma^{\prime}\right|=n<r p$. Then $\eta^{\prime}$ is a chain map because (the sum ranges over the set of faces $u$ of an $n$-cube):

$$
\begin{aligned}
\eta^{\prime} \partial\left(\sigma, \sigma^{\prime}\right) & =\sum \pm \eta^{\prime}\left(\sigma \circ u, \sigma^{\prime} \circ u\right) \\
& =\sum \pm\left((\sigma \circ u) \otimes\left(\sigma^{\prime} \circ u\right)\right) \square \Phi^{r} \gamma\left(x^{n-1}\right) \\
& =\sum \pm\left(\sigma \otimes \sigma^{\prime}\right) \square\left(h_{u} \otimes h_{u}\right) \Phi^{r} \gamma\left(x^{n-1}\right) \\
& =\sum \pm\left(\sigma \otimes \sigma^{\prime}\right) \square \Phi^{r} h_{u} \gamma\left(x^{n-1}\right) \\
& =\sum \pm\left(\sigma \otimes \sigma^{\prime}\right) \square \Phi^{r} \gamma(u) \\
& =\left(\sigma \otimes \sigma^{\prime}\right) \square \Phi^{r} D^{r} \gamma\left(x^{n}\right) \\
& =\left(\sigma \otimes \sigma^{\prime}\right) \square D^{r} \Phi^{r} \gamma\left(x^{n}\right) \\
& =\partial\left(\left(\sigma \otimes \sigma^{\prime}\right) \square \Phi^{r} \gamma\left(x^{n}\right)\right)=\partial \eta^{\prime}\left(\sigma, \sigma^{\prime}\right) .
\end{aligned}
$$

Similarly one verifies that $F^{\prime}$ is a derivation homotopy from $\eta$ to $\eta^{\prime}$.

Put $F, \phi: \mathrm{CU}_{*}^{r}(X) \rightarrow \mathrm{CU}_{*}^{r}(X) \otimes \mathrm{CU}_{*}^{r}(X)$ by $F=F^{\prime} \circ \mathrm{CU}_{*}^{r}(\Delta), \phi=\eta^{\prime} \circ$ $\mathrm{CU}_{*}^{r}(\Delta)$. Thus,

$$
\phi(\sigma)=(\sigma \otimes \sigma) \square \Phi^{r} \gamma\left(x^{n}\right)
$$

Notice that

$$
\pi_{1} \eta^{\prime}=\mathrm{CU}_{*}^{r}\left(p_{X}\right), \quad \pi_{2} \eta^{\prime}=\mathrm{CU}_{*}^{r}\left(p_{Y}\right),
$$

$p_{X}$ and $p_{Y}$ denoting the projections of $X \times Y$ onto its factors, so that $\pi_{1} \phi=$ $\pi_{2} \phi=1$. It is now straightforward to verify

Proposition 7.4. Suppose $R \supseteq \mathbf{Q}, r \geq 1$. Then $\phi$ is a functorial cocommutative coassociative dga homomorphism, and $F$ is a functorial derivation homotopy from $\psi$ to $\phi$. In particular, $\phi$ is a cocommutative coassociative diagonal approximation on $\mathrm{CU}_{*}^{r}()$ for topological monoids. Furthermore, the functorial chain equivalence $\eta^{\prime}$ is cocommutative and coassociative in the following sense. If $t: X \times Y \rightarrow Y \times X$ switches coordinates, then $\eta^{\prime} \circ \mathrm{CU}_{*}^{r}(t)=\tau \eta^{\prime}$, and $\left(\eta_{(X \times Y)}^{\prime} \otimes 1\right) \eta_{(X \times Y) \times Z}^{\prime}=\left(1 \otimes \eta_{(Y \times Z)}^{\prime}\right) \eta_{X \times(Y \times Z)}^{\prime}: \mathrm{CU}_{*}^{r}(X \times Y \times Z) \rightarrow \mathrm{CU}_{*}^{r}(X) \otimes$ $\mathrm{CU}_{*}^{r}(Y) \otimes \mathrm{CU}_{*}^{r}(Z)$.

Theorem 7.5. Suppose $R \supseteq \mathbf{Q}, r \geq 1$. There is a functor

$$
\mathscr{E}: \mathrm{MON}_{r} \rightarrow \mathrm{DGL}_{r}(R)
$$


such that $U \mathscr{E}=\mathrm{CU}_{*}^{r}()$. Furthermore, the coproduct $\Delta_{\mathscr{E}(X)}$ on $U \mathscr{E}(X)$, with respect to which each $w \in \mathscr{E}(X)$ is primitive, is a diagonal approximation.

Proof. It suffices to check this when $R=\mathbf{Q}$, since we may then tensor over Q with $R$. Put $\mathscr{E}(X)=\operatorname{ker}(\bar{\phi})$. Then $\mathscr{E}$ is a functor, and by [10] we have $U \mathscr{E}=\mathrm{CU}_{*}^{r}()$ and $\phi=\Delta_{\mathscr{E}(X)}$.

Theorem 7.6. Suppose $p=\rho(R)<\infty$. Then Proposition 7.4 and Theorem 7.5 hold, up to dimension $r p-1$. For $X, Y \in \mathrm{MON}_{r}$, there is a functorial cocommutative coassociative

$$
\eta^{\prime}: \mathrm{CU}_{<r p}^{r}(X \times Y) \rightarrow\left(\mathrm{CU}_{*}^{r}(X) \otimes \mathrm{CU}_{*}^{r}(Y)\right)_{<r p}
$$

and a functorial cocommutative coassociative "diagonal"

$$
\phi: \mathrm{CU}_{<r p}^{r}(X) \rightarrow\left(\mathrm{CU}_{*}^{r}(X) \otimes \mathrm{CU}_{*}^{r}(X)\right)_{<r p} .
$$

There are natural chain homotopies between $\eta$ and $\eta^{\prime}$, and between $\psi$ and $\phi$, so $\phi$ serves as a diagonal approximation below dimension $r p$. There is a functor $\mathscr{E}: \mathrm{MON}_{r} \rightarrow \operatorname{DGL}(R)$ such that $(U \mathscr{E})_{<r p}()=\mathrm{CU}_{<r p}^{r}()$, for which $\Delta_{\mathscr{E}(X)}=\phi$.

We discuss next the relationship between the functor $\mathscr{E}$ of Theorem 7.5 and various functors that occur in rational homotopy theory. Two functors from a category $\mathscr{X}$ to a category consisting of chain complexes are quasi-isomorphic if there is a natural transformation between them (going either way) which is a quasi-isomorphism for each object of $\mathscr{X}$. Two such functors $\mathscr{F}$ and $\mathscr{F}^{\prime}$ are weakly equivalent, denoted $\mathscr{F} \cong \mathscr{F}^{\prime}$, if there is a sequence $\mathscr{F}=\mathscr{F}_{0}$, $\mathscr{F}_{1}, \ldots, \mathscr{F}_{m}=\mathscr{F}^{\prime}$ of functors with $\mathscr{F}_{i}$ quasi-isomorphic to $\mathscr{F}_{i-1}$.

Recall the bar and cobar functors

$$
\mathrm{DGA} \underset{\mathrm{B}}{\stackrel{B}{\rightleftarrows}} \mathrm{DGcoA},
$$

where $\mathrm{DG} c \mathrm{~A}$, or $\mathrm{DG} \operatorname{coA}(R)$, denotes the category of differential graded coassociative 1-connected coalgebras over $R$. In [13] Quillen discussed the functor

$$
C: \mathrm{DGL} \rightarrow \mathrm{CDG} \operatorname{coA},
$$

where $\mathrm{CDGcoA}$ is the full subcategory of $\mathrm{DGcoA}$ consisting of cocommutative coalgebras. We have the relationships

$$
B \mathrm{~B} \cong \mathrm{id}, \quad \mathrm{B} B \cong \mathrm{id}, \quad B U \cong C, \quad \mathrm{~B} C \cong U .
$$

Let TOP $_{r}$ denote the category of $r$-connected topological spaces. The Moore loop space functor, denoted $\Omega$, carries $\mathrm{TOP}_{r}$ to $\mathrm{MON}_{r}$. Adams [1] showed that $\mathrm{B} C_{*}^{2} \cong \mathrm{CU}_{*}^{1} \Omega$ as functors from $\mathrm{TOP}_{1}$ to DGA, where $C_{*}^{2}$ denotes the 1-connected Eilenberg subcomplex of $C_{*}$. Implicit in his paper is the slight generalization that

$$
\mathrm{B} C_{*}^{2} \cong \mathrm{CU}_{*}^{r} \Omega: \mathrm{TOP}_{r} \rightarrow \mathrm{DGA}
$$

for any $r \geq 1$. 
Proposition 7.7. For $r \geq 1, R \supseteq \mathbf{Q}$, let $\mathscr{E}$ be as in Proposition 7.4. There is a weak equivalence of functors

$$
C_{*}^{2} \cong C \mathscr{E} \Omega: \mathrm{TOP}_{r} \rightarrow \mathrm{DGcoA}(R),
$$

and $C \mathscr{E} \Omega(X)$ lies in $\mathrm{CDG} \operatorname{coA}(R)$.

Proof. By (30) and (31) and Theorem 7.5, we have

$$
C_{*}^{2} \cong B \mathrm{~B} C_{*}^{2} \cong B\left(\mathrm{CU}_{*}^{r}\right) \Omega=B U \mathscr{E} \Omega \cong C \mathscr{E} \Omega \text {. }
$$

As we suggested in the introduction, the functor $\mathscr{E} \Omega$ coincides up to homotopy with the rational homotopy theory functor

$$
Q: \operatorname{TOP}_{1} \rightarrow \operatorname{DGL}(\mathbf{Q})
$$

introduced by Quillen [12]. This is proved in the forthcoming paper [6], where a short list of axioms is given which provides a necessary and sufficient condition for any such functor to be weakly equivalent to Quillen's $Q$. In particular, since $Q$ is a complete rational homotopy invariant, so is $\mathscr{E} \Omega$.

\section{The Adams-Hilton model as a HaH}

We review the properties of the Adams-Hilton model $\mathbf{A}(X)$ for a simplyconnected CW complex $X$. We show that $\mathbf{A}(X)$ is an $r$-mild Hah when $X$ is $r$-connected and $\operatorname{dim}(X) \leq r \rho(R)$. Applying Theorem 4.8, we obtain an $r$-mild $\operatorname{dgL} \mathbf{L}(X)$ associated to $X$. We then explore the properties of $\mathbf{L}$ as a model.

As always, $p=\rho(R)$ for some commutative ring with unity $R$. Let $\mathrm{CW}_{r}$ (resp. $\mathrm{CW}_{r}^{k}$ ) be the full subcategory of $\mathrm{TOP}_{r}$ consisting of $\mathrm{CW}$ complexes with trivial $r$-skeleton (resp. and of dimension $\leq k$ ). Adams and Hilton [2] constructed, for each $X \in \mathrm{CW}_{1}$, a dga over $R \mathbf{A}(X)=\left(A(X), d_{X}\right)$. The dga $\mathbf{A}(X)$ comes with a quism $\theta_{X}: \mathbf{A}(X) \rightarrow \mathrm{CU}_{*}^{1}(\Omega X)$. Since arbitrary choices are made in the construction of $d_{X}$ and $\theta_{X}$, and $\mathbf{A}(X)$ depends upon our choice of CW decomposition for $X$, we do not think of $\mathbf{A}(X)$ as being unique.

Likewise, for any (not necessarily cellular) map $f: X \rightarrow Y$ in $\mathrm{CW}_{1}$ and for any choice of models $\left(\mathbf{A}(X), \theta_{X}\right)$ and $\left(\mathbf{A}(Y), \theta_{Y}\right)$, Adams and Hilton constructed a DGA homomorphism $\mathbf{A}(f): \mathbf{A}(X) \rightarrow \mathbf{A}(Y)$. The homomorphism $\mathbf{A}(f)$ comes with a derivation homotopy $\psi_{f}$ from $\left(\mathrm{CU}_{*}^{1} \Omega f\right) \circ \theta_{X}$ to $\theta_{Y} \circ \mathbf{A}(f)$. Again, both $\mathbf{A}(f)$ and $\psi_{f}$ require us to make some arbitrary choices, so we do not view them as unique. We call $\left(\mathbf{A}(f), \psi_{f}\right)$ a model for $f$.

We list now fourteen major properties of the model $\mathbf{A}$. Theorem 8.1 is nearly a complete summary of results for the Adams-Hilton paper [2].

Theorem 8.1 (Adams-Hilton). The model $\mathbf{A}: \mathrm{CW}_{1} \rightarrow \operatorname{DGA}(R)$ has the following properties.

(a) If $X=(\mathrm{pt}) \cup\left(\cup_{\alpha \in S} e_{\alpha}\right)$ is a cell decomposition for $X,\left|e_{\alpha}\right| \geq 2$, then the underlying algebra $A(X)$ is isomorphic to $R\left\langle b_{\alpha} \mid \alpha \in S\right\rangle$, where $\left|b_{\alpha}\right|=\left|e_{\alpha}\right|-1$. Moreover, the linearized chain complex $\mathbf{A}(X)_{+} /\left(\mathbf{A}(X)_{+}\right)^{2}$ may be identified with the desuspension of the reduced cellular chain complex for $X$. 
(b) In particular, if $X \in \mathrm{CW}_{r}$, then $\mathbf{A}(X)$ is r-reduced; if $X \in \mathrm{CW}_{r}^{r p}$, then $\mathbf{A}(X) \in \mathrm{DGA}_{r}(R)$.

(c) If $f \simeq g$, then $\mathbf{A}(f) \simeq \mathbf{A}(g)$ in DGA. In particular, two models for the same map must be homotopic in DGA. Conversely, if $\zeta \simeq \mathbf{A}(f)$ in $D G A$, then there exists a derivation homotopy $\psi^{\prime}$ such that $\left(\zeta, \psi^{\prime}\right)$ is also a model for $f$.

(d) One model for the identity map $1_{X}$ is $\left(1_{\mathbf{A}(X)}, 0\right)$.

(e) If $X \stackrel{f}{\rightarrow} Y \stackrel{g}{\rightarrow} Z$, then $\left(\mathbf{A}(g f), \psi_{g f}\right)$ may be taken to be $(\mathbf{A}(g) \circ \mathbf{A}(f)$, $\left.\mathrm{CU}_{*}^{1}(\Omega g) \circ \psi_{f}+\psi_{g} \circ \mathbf{A}(f)\right)$.

(f) If $f: X \rightarrow Y$ is a homotopy equivalence, so is $\mathbf{A}(f)$.

(g) Let $X_{0}$ be a subcomplex of $X$, say $X=(\mathrm{pt}) \cup\left(\cup_{\alpha \in S} e_{\alpha}\right), X_{0}=(\mathrm{pt}) \cup$ $\left(\bigcup_{\alpha \in S_{0}} e_{\alpha}\right), S_{0} \subseteq S$. Given any model $\left(A\left(X_{0}\right), d_{X_{0}}, \theta_{X_{0}}\right)$, there is a model $\left(A(X), d_{X}, \theta_{X}\right)$ for which $d_{X}$ and $\theta_{X}$ are extensions over $A(X)$ of $d_{X_{0}}$ and $\theta_{X_{0}}$.

(h) Under the hypotheses of (g), let $f: X \rightarrow Y$ be a map and put $f_{0}=\left.f\right|_{X_{0}}$. Given any models $\left(\mathbf{A}(Y), \theta_{Y}\right)$ and $\left(\mathbf{A}\left(f_{0}\right), \psi_{f_{0}}\right)$, there is a model $\left(\mathbf{A}(f), \psi_{f}\right)$ for which $\mathbf{A}(f)$ and $\psi_{f}$ are extensions over $A(X)$ of $\mathbf{A}\left(f_{0}\right)$ and $\psi_{f_{0}}$.

(i) Let $\left\{X_{\beta}\right\} \subseteq \mathrm{CW}_{1}$ be a family of subcomplexes of a $C W$ complex $X$, and suppose $X=\bigcup_{\beta} X_{\beta}$. Suppose we have models $\left(\mathbf{A}\left(X_{\beta}\right), \theta_{X_{\beta}}\right)$ satisfying the coherency conditions

$$
\begin{gathered}
\left.d_{X_{\beta}}\right|_{A\left(X_{\beta} \cap X_{\gamma}\right)}=\left.d_{X_{\gamma}}\right|_{A\left(X_{\beta} \cap X_{\gamma}\right)}, \\
\left.\theta_{X_{\beta}}\right|_{A\left(X_{\beta} \cap X_{\gamma}\right)}=\left.\theta_{X_{y}}\right|_{A\left(X_{\beta} \cap X_{y}\right)}
\end{gathered}
$$

for each pair of indices $(\beta, \gamma)$. Then $\operatorname{colim}\left\{\mathbf{A}\left(X_{\beta}\right), \theta_{X_{\beta}}\right\}$ is an Adams-Hilton model for $X$.

(j) Under the hypotheses of (i), let $f: X \rightarrow Y$ be a map and put $f_{\beta}=\left.f\right|_{X_{\beta}}$. Fixing a model $\left(\mathbf{A}(Y), \theta_{Y}\right)$, suppose we have models $\left(\mathbf{A}\left(f_{\beta}\right), \psi_{f_{\beta}}\right)$ satisfying the coherency conditions

$$
\begin{gathered}
\left.\mathbf{A}\left(f_{\beta}\right)\right|_{A\left(X_{\beta} \cap X_{\gamma}\right)}=\left.\mathbf{A}\left(f_{\gamma}\right)\right|_{A\left(X_{\beta} \cap X_{\gamma}\right)}, \\
\left.\psi_{f_{\beta}}\right|_{A\left(X_{\beta} \cap X_{\gamma}\right)}=\left.\psi_{f_{\gamma}}\right|_{A\left(X_{\beta} \cap X_{\gamma}\right)} .
\end{gathered}
$$

Then $\operatorname{colim}\left\{\mathbf{A}\left(f_{\beta}\right), \psi_{f_{\beta}}\right\}$ is an Adams-Hilton model for $f$.

(k) Let $f_{0}: S^{n} \rightarrow X_{0}, n \geq 2$, and extend $f_{0}$ to $f: D^{n+1} \rightarrow X=X_{0} \cup_{f_{0}} e^{n+1}$. Choosing the standard two-cell decomposition of $D^{n+1}$ we have $A\left(D^{n+1}\right)=$ $R\left\langle z, z_{0}\right\rangle$ with $\left|z_{0}\right|=n-1,|z|=n, d(z)=-z_{0}$. Let $\left(\mathbf{A}\left(X_{0}\right), \theta_{X_{0}}\right)$ and $\left(\mathbf{A}\left(f_{0}\right), \psi_{f_{0}}\right)$ be models for $X_{0}$ and $f_{0}$. Then one for $X$ is given by $A(X)=A\left(X_{0}\right) \amalg R\left\langle b_{f}\right\rangle,\left|b_{f}\right|=n ; d_{X}(x)=d_{X_{0}}(x)$ for $x \in A\left(X_{0}\right), d_{X}\left(b_{f}\right)$ $=-\mathbf{A}\left(f_{0}\right)\left(z_{0}\right) ; \theta_{X}(x)=\theta_{X_{0}}(x)$ for $x \in A\left(X_{0}\right), \theta_{X}\left(b_{f}\right)=\operatorname{CU}_{*}^{1}(\Omega f)\left(\theta_{D^{n+1}}(z)\right)+$ $\psi_{f_{0}}\left(z_{0}\right)$.

(1) Under the hypotheses of $(\mathbf{k})$, let $g_{0}: X_{0} \rightarrow Y_{0}$ have model $\left(\mathbf{A}\left(g_{0}\right), \psi_{g_{0}}\right)$. Extend $g_{0}$ to $g: X \rightarrow Y=Y_{0} \cup_{g_{0} f_{0}} e^{n+1}$ in the obvious way, and choose models 
for $X$ and for $Y$ as in $(\mathbf{k})$. Then an extension $\left(\mathbf{A}(g), \psi_{g}\right)$ of $\left(\mathbf{A}\left(g_{0}\right), \psi_{g_{0}}\right)$ may be chosen for which $\mathbf{A}(g)\left(b_{f}\right)=\left(b_{g f}\right), \psi_{g}\left(b_{f}\right)=0$.

Two further properties concern the model for a product space $X \times Y$. If $X=(\mathrm{pt}) \cup\left(\bigcup_{\alpha \in S} e_{\alpha}\right)$ and $Y=(\mathrm{pt}) \cup\left(\bigcup_{\alpha \in S^{\prime}} e_{\alpha}\right)$, then $A(X \times Y)$ is freely generated as an $R$-algebra by $\left\{b_{\alpha} \mid \alpha \in S^{\prime \prime}\right\}$, where

$$
S^{\prime \prime}=S \cup S^{\prime} \cup\left(S \times S^{\prime}\right) .
$$

(Adams and Hilton point out that $X \times Y$ need not be a $C W$ complex for their construction to exist.) We define

$$
\nu=\nu_{X Y}: A(X \times Y) \rightarrow A(X) \otimes A(Y)
$$

by $\nu\left(b_{\alpha}\right)=b_{\alpha} \otimes 1$ for $\alpha \in S, \nu\left(b_{\alpha}\right)=1 \otimes b_{\alpha}$ for $\alpha \in S^{\prime}, \nu\left(b_{\alpha}\right)=0$ for $\alpha \in\left(S \times S^{\prime}\right)$.

(m) Given $\left(\mathbf{A}(X), \theta_{X}\right)$ and $\left(\mathbf{A}(Y), \theta_{Y}\right)$, it is possible to choose $d_{X \times Y}$ and $\theta_{X \times Y}$ for $A(X \times Y)$ such that $\nu_{X Y}$ is a DGA homomorphism and the following diagram commutes up to homotopy:

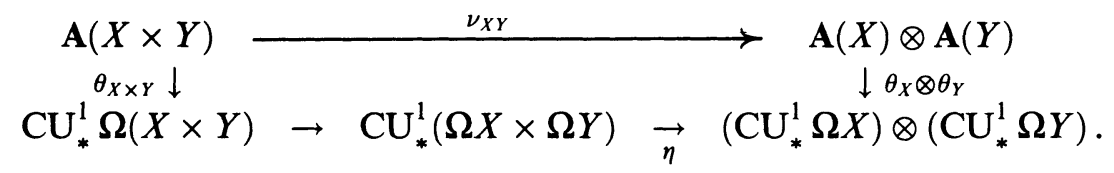

Furthermore, letting $X \stackrel{p_{X}}{\leftarrow} X \times Y \stackrel{p_{Y}}{\rightarrow} Y$ denote the projections, we may take $\mathbf{A}\left(p_{X}\right)=\pi_{1} \nu_{X Y}$ and $\mathbf{A}\left(p_{Y}\right)=\pi_{2} \nu_{X Y}$.

(n) Given maps $f: X_{0} \rightarrow X$ and $g: Y_{0} \rightarrow Y$ and models for $f$ and $g$, any model $\left(\mathbf{A}(f \times g), \psi_{f \times g}\right)$ for $f \times g$ makes the following diagram commute up to homotopy:

$$
\begin{array}{ccc}
\mathbf{A}\left(X_{0} \times Y_{0}\right) & \underset{\nu_{X_{0} Y_{0}}}{\longrightarrow} & \mathbf{A}\left(X_{0}\right) \otimes \mathbf{A}\left(Y_{0}\right) \\
\mathbf{A}(f \times g) \downarrow & & \downarrow \mathbf{A}(f) \otimes \mathbf{A}(g) \\
\mathbf{A}(X \times Y) & \underset{\nu_{X Y}}{\mathbf{A}}(X) \otimes \mathbf{A}(Y) .
\end{array}
$$

Remark. If we restrict ourselves to spaces and maps in $\mathrm{CW}_{r}$, then we may presume that $\theta_{X}: \mathbf{A}(X) \rightarrow \mathrm{CU}_{*}^{r}(\Omega X)$, and likewise for $\psi_{r}$.

Notation. Let $\Delta: X \rightarrow X \times X$ be the diagonal map, and let $\lambda_{X}$ be the composite.

$$
\lambda_{X}=\nu_{X X} \circ \mathbf{A}(\Delta): \mathbf{A}(X) \rightarrow \mathbf{A}(X) \otimes \mathbf{A}(X) .
$$

Lemma 8.2. With $\psi$ as in (21), the following diagram commutes up to homotopy:

$$
\begin{array}{cccc}
\mathbf{A}(X) & \stackrel{\lambda_{X}}{\longrightarrow} & \mathbf{A}(X) \otimes \mathbf{A}(X) \\
\theta_{X} \downarrow & & \downarrow \theta_{X} \otimes \theta_{X} \\
\mathrm{CU}_{*}^{1} \Omega X & \longrightarrow & \left(\mathrm{CU}_{*}^{1} \Omega X\right) \otimes\left(\mathrm{CU}_{*}^{1} \Omega X\right) .
\end{array}
$$

Proof. Choose $d_{X \times X}$ and $\theta_{X \times X}$ according to Theorem $8.1(\mathrm{~m})$. 
Proposition 8.3. The model $\mathbf{A}()$, together with $\lambda_{()}$, takes $\mathrm{CW}_{1}\left(\right.$ resp. $\left.\mathrm{CW}_{r}^{r p}\right)$ to $\mathrm{HAH}(R)$ (resp. $\left.\mathrm{HAH}_{r}(R)\right)$.

Proof. The four homotopies for $\lambda_{X}$ follow from Proposition 7.1, Lemma 8.2, and the lifting lemma. When $f: X \rightarrow Y$, the diagram

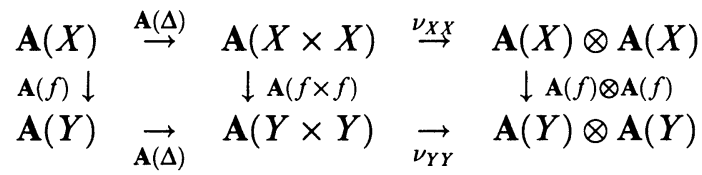

commutes up to homotopy by Theorem $8.1(\mathrm{c}, \mathrm{n})$.

Construction 8.4. For $X \in \mathrm{CW}_{r}^{r p},\left(\mathbf{A}(X), \lambda_{X}\right)$ is an $r$-mild Hah. Applying Theorem 5.6 yields an $r$-mild $\operatorname{dgL} \mathbf{L}(X)=\left(L(X), \delta_{X}\right)$ for which $U \mathbf{L}(X) \approx$ $\mathbf{A}(X)$ and $\Delta_{L(X)} \simeq \lambda_{X}$. For any map $f: X \rightarrow Y$ in $\mathrm{CW}_{r}^{r p}$, we have by Theorem 6.4 a DGL homomorphism $\mathbf{L}(f): \mathbf{L}(X) \rightarrow \mathbf{L}(Y)$ such that $U \mathbf{L}(f) \simeq \mathbf{A}(f)$. We view $\mathbf{L}$ as a model from $\mathrm{CW}_{r}^{r p}$ to $\operatorname{DGL}_{r}(R)$. We view $\mathbf{L}(X)$ as coming with a quism $\theta_{X}: U \mathbf{L}(X) \rightarrow \mathrm{CU}_{*}^{r}(\Omega X)$, and $\mathbf{L}(f)$ as coming with a derivation homotopy $\psi_{f}$ from $\mathrm{CU}_{*}^{r} \Omega f \circ \theta_{X}$ to $\theta_{Y} \circ U \mathbf{L}(f)$.

For completeness we list the properties of $\mathbf{L}$, since they are likely to be useful in applications.

Theorem 8.5. The model $\mathbf{L}: \mathrm{CW}_{r}^{r p} \rightarrow \mathrm{DGL}_{r}(R)$ has the following properties.

(a) If $X=(\mathrm{pt}) \cup\left(\cup_{\alpha \in S} e_{\alpha}\right)$ is a cell decomposition for $X, r p \geq\left|e_{\alpha}\right|>r$, then the underlying Lie algebra $\mathbf{L}(X)$ is isomorphic to $\mathrm{L}_{R}\left\langle b_{\alpha} \mid \alpha \in S\right\rangle$, where $\left|b_{\alpha}\right|=\left|e_{\alpha}\right|-1$. The linearization $\mathbf{L}(X) /[\mathbf{L}(X), \mathbf{L}(X)]$ may be identified with the desuspension of the reduced cellular chain complex for $X$.

(b) $\left(U \mathbf{L}(X), \theta_{X}\right)\left(\right.$ resp. $\left.\left(U \mathbf{L}(f), \psi_{f}\right)\right)$ is an Adams-Hilton model for $X$ (resp. $f)$. In particular, $\theta_{X}$ induces an isomorphism of $R$-algebras

$$
\left(\theta_{X}\right)_{*}: H_{*}(U \mathbf{L}(X)) \stackrel{\approx}{\rightarrow} H_{*}(\Omega X ; R) .
$$

If $R$ is a field, (35) is an isomorphism of Hopf algebras.

(c) If $f \simeq g$, then $\mathbf{L}(f) \simeq \mathbf{L}(g)$. In particular, two models for the same map must be homotopic. Conversely, if $\zeta \simeq L(f)$ in $\operatorname{DGL}_{r}(R)$, then there exists a derivation homotopy $\psi^{\prime}$ such that $\left(\zeta, \psi^{\prime}\right)$ is also a model for $f$.

(d) One model for the identity map $1_{X}$ is $\left(1_{L(X)}, 0\right)$.

(e) If $X \stackrel{f}{\rightarrow} Y \stackrel{g}{\rightarrow} Z$, then $\left(\mathbf{L}(g f), \psi_{g f}\right)$ may be taken to be $(\mathbf{L}(g) \circ \mathbf{L}(f)$, $\left.\mathrm{CU}_{*}^{r}(\Omega g) \circ \psi_{f}+\psi_{g} \circ U \mathbf{L}(f)\right)$.

(f) If $f: X \rightarrow Y$ is a homotopy equivalence, so is $L(f)$.

(g)-(1) Analogs of Theorem $8.1(\mathrm{~g})-(\mathrm{l})$ are true, with $\mathrm{CW}_{r}^{r p}, \mathrm{~L}, L, \delta_{X}$ everywhere replacing $\mathrm{CW}_{1}, \mathbf{A}, A, d_{X}$. For (k) and (1), require that $r p>n>r$.

Proof. (a)-(f) are straightforward, as are (g)-(l) once we make a certain observation. The observation is that the constructions in Theorems 5.6 and 6.3 are natural with respect to certain sub-Hah's. Suppose in Theorem 5.6 that the Hah $(A, d, \psi)$ satisfies $A=T(V \oplus W)$ and $d(V) \subseteq T V$ and $\psi(V) \subseteq T V \otimes T V$, so 
$\left(T V,\left.d\right|_{T V},\left.\psi\right|_{T V}\right)$ is also a Hah. Applying the construction of Theorem 5.6 directly to this sub-Hah yields a sequence $\left\{\tilde{\psi}^{(n)}\right\}$ of homotopic coproducts whose limit or $(r p-1)$ st term $\tilde{\psi}$ is cocommutative and coassociative on $T V$. Some arbitrary choices may be involved where the homotopy extension property is used. Fixing any such set of choices, it is possible to perform the construction of Theorem 5.6 on the whole $(A, d, \psi)$ in such a way that each term of the resulting sequence $\left\{\psi^{(n)}\right\}$ satisfies $\left.\psi^{(n)}\right|_{T V}=\tilde{\psi}^{(n)}$. Thus the limit or $(r p-1)$ st term of $\left\{\psi^{(n)}\right\}$ restricts to $\tilde{\psi}$. Because of this, $\mathbf{L}$ easily inherits the various coherency properties from those of $\mathbf{A}$.

Corresponding to Theorem $8.1(\mathrm{~m})$ and $(\mathrm{n})$, we have

Theorem 8.6. Let $X, Y \in \mathrm{CW}_{r}^{r p}$. Suppose $\operatorname{dim}(X)+\operatorname{dim}(Y) \leq r p$. There is $a$ quism

$$
\xi_{X Y}: \mathbf{L}(X \times Y) \rightarrow \mathbf{L}(X) \oplus \mathbf{L}(Y)
$$

such that the diagram

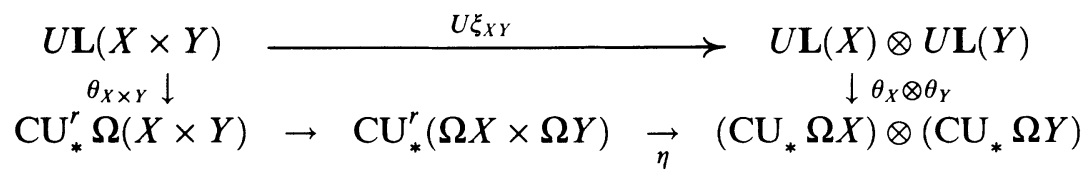

commutes up to homotopy. The first and second components of $\xi_{X Y}$ are homotopic to $\mathbf{L}\left(p_{X}\right)$ and $\mathbf{L}\left(p_{Y}\right)$, respectively. Furthermore, $\xi$ is natural in the following sense. Given any maps $f: X_{0} \rightarrow X$ and $g: Y_{0} \rightarrow Y$ in $\mathrm{CW}_{r}^{r p}$ where $\operatorname{dim}\left(X_{0}\right)+\operatorname{dim}\left(Y_{0}\right) \leq r p$, the following diagram commutes up to homotopy:

$$
\begin{array}{ccc}
\mathbf{L}\left(X_{0} \times Y_{0}\right) & \underset{\xi_{X_{0} Y_{0}}}{\rightarrow} & \mathbf{L}\left(X_{0}\right) \oplus \mathbf{L}\left(Y_{0}\right) \\
\mathbf{L}(f \times g) \downarrow & & \downarrow \mathbf{L}(f) \oplus \mathbf{L}(g) \\
\mathbf{L}(X \times Y) & \overrightarrow{\xi_{X Y}} & \mathbf{L}(X) \oplus \mathbf{L}(Y)
\end{array}
$$

In particular, when $2 \cdot \operatorname{dim}(X) \leq r p$, the diagonal map $\triangle: X \rightarrow X \times X$ has a model $\mathbf{L}(\Delta)$ such that $\xi_{X X} \circ \mathbf{L}(\Delta)=\tilde{\Delta}: \mathbf{L}(X) \rightarrow \mathbf{L}(X) \oplus \mathbf{L}(X)$, where $\tilde{\Delta}(x)=$ $(x, x)$.

Proof. By Theorem $8.1(\mathrm{~m})$ we have the quism

$$
\nu_{X Y}: U \mathbf{L}(X \times Y) \stackrel{\cong}{\rightarrow} U \mathbf{L}(X) \otimes U \mathbf{L}(Y)=U(\mathbf{L}(X) \oplus \mathbf{L}(Y)) .
$$

By Theorem 6.3, we know that $\nu_{X Y} \simeq U \xi_{X Y}$ for some quism $\xi_{X Y}: \mathbf{L}(X \times Y) \rightarrow$ $\mathbf{L}(X) \oplus \mathbf{L}(Y)$. The Lemaire-Aubry theorem yields $\xi_{X Y} \simeq\left(\mathbf{L}\left(p_{X}\right), \mathbf{L}\left(p_{Y}\right)\right)$. By Theorem $8.1(\mathrm{~m})$, the commutativity up to homotopy of $(36)$ is immediate. Theorem 8.1(n) together with the Lemaire-Aubry theorem shows that (37) commutes up to homotopy. As to $\xi_{X X} \circ \mathbf{L}(\Delta)$, note that $U\left(\xi_{X X} \circ \mathbf{L}(\Delta)\right)=\nu_{X X} \circ$ $U \mathbf{L}(\Delta)=\lambda_{X} \simeq \Delta_{L(X)}=U(\tilde{\Delta})$, so Lemaire-Aubry shows that $\tilde{\Delta} \simeq \xi_{X X} \circ \mathbf{L}(\Delta)$. Now use the lifting lemma ( $\xi_{X X}$ is a surjective quism) to replace $\mathbf{L}(\Delta)$ by a homotopic homomorphism $\zeta$ for which $\xi_{X X} \circ \zeta=\tilde{\Delta}$. 
Proposition 8.7. (a) There is a weak equivalence

$$
C_{*}^{2} \cong C \mathbf{L}: \mathrm{CW}_{r}^{r p} \rightarrow \mathrm{DG} \operatorname{coA}(R),
$$

and $C \mathbf{L}(X)$ lies in $\operatorname{CDG} \operatorname{coA}(R)$.

(b) On TOP ${ }_{r}$ we may construct "commutative cochains" over $R$ up to dimension $r p$.

Proof. (a) Imitate the proof of Proposition 7.7, with $\mathbf{L}$ replacing $\mathscr{E} \Omega$.

(b) Given any space $X \in \mathrm{TOP}_{r}$, choose an $r$-connected $\mathrm{CW}$ approximation and take its $r p$-skeleton, i.e., $f: Y \rightarrow X$ with $Y \in \mathrm{CW}_{r}^{r p}$. Then $C_{*}^{2}(X) \underset{f_{*}}{\stackrel{\simeq}{\leftrightarrows}}$ $C_{*}^{2}(Y) \simeq C \mathbf{L}(Y)$ in dimensions $\leq r p$.

Theorem 8.8. Suppose $R \supseteq \mathbf{Q}$, and let $\phi$ be as in Proposition 7.4. It is possible to choose $\theta_{X}$ such that $\theta_{X}(\mathbf{L}(X)) \subseteq \operatorname{ker}(\bar{\phi})$. The restriction of $\theta_{X}$ to $\mathbf{L}(X)$ determines a DGL quism

$$
\omega_{X}: \mathbf{L}(X) \rightarrow \mathscr{E} \Omega(X),
$$

which is natural in the sense that there is a DGL homotopy $\omega_{Y} \circ \mathbf{L}(f) \simeq \mathscr{E} \Omega(f) \circ$ $\omega_{X}: \mathbf{L}(X) \rightarrow \mathscr{E} \Omega(Y)$ for any map $f: X \rightarrow Y$.

Proof. Applying Theorem 6.3 to the HAH homomorphism $\theta_{X}: U \mathbf{L}(X) \rightarrow$ $\left(\mathrm{CU}_{*}^{r} \Omega X, \phi\right)$, we obtain a HAH homomorphism $\theta_{X}^{\prime}$ homotopic to $\theta_{X}$ for which $\theta_{X}^{\prime}(\mathbf{L}(X)) \subseteq \operatorname{ker}(\bar{\phi})$. Since any DGA homomorphism homotopic to $\theta_{X}$ could also serve as a valid choice for $\theta_{X}$, we may replace $\theta_{X}$ by $\theta_{X}^{\prime}$. We have proved the first claim and the existence of $\omega_{X}$. The naturality follows easily from the Lemaire-Aubry theorem.

Remark. If we weaken the concept of weak equivalence of functors to allow for models as well as functors and to allow for quisms which are natural transformations only up to homotopy, Theorem 8.8 tells us when $R \supseteq \mathbf{Q}$ that $\mathbf{L} \cong$ $\mathscr{E} \Omega \cong Q$. Also, the " $\mathbf{L}$ " constructed for the ring $R=\mathbf{Z}[1 /(p-1) !]$, call it $\mathbf{L}^{R}$, and the " $\mathbf{L}$ " constructed for $\mathbf{Q}$, call it $\mathbf{L}^{\mathbf{Q}}$, are related via $\mathbf{L}^{\mathbf{Q}}()=\mathbf{L}^{R}() \otimes \mathbf{Q}$. Thus $\mathbf{L}^{R}() \otimes \mathbf{Q} \cong Q$, as needed in diagram (1).

\section{THE MOD $p$ COHOMOLOGY OF LOOP SPACES}

In this final section we will prove Wilkerson's conjecture [9]. According to this conjecture, if $X$ is a finite simply-connected CW complex, then for $p \gg 0$ $p$ th powers vanish in $\widetilde{H}^{*}\left(\Omega X ; \mathbf{Z}_{p}\right)$.

Theorem 9.1. Let $p$ be a prime and let $X$ be an r-connected $C W$ complex of dimension $n$, where $r p \geq n$. Then (a) pth powers vanish in $\widetilde{H}^{*}\left(\Omega X ; \mathbf{Z}_{p}\right)$, and (b) $H_{*}\left(\Omega X ; \mathbf{Z}_{p}\right)$ is primitively generated as a $\mathbf{Z}_{p}$-algebra.

Proof. The equivalence of Theorem 9.1(a) and (b) is given in [10, Proposition 4.20]. The theorem follows at once from Theorem 8.5(b) and the following lemma. 
Lemma 9.2. Let $(L, \delta)$ be a dgL over $\mathbf{Z}_{p}$. Then the Hopf algebra $H_{*} U(L, \delta)$ is primitively generated as a $\mathbf{Z}_{p}$-algebra.

Proof. Because $U L$ is primitively generated, $p$ th powers vanish in the dual Hopf algebra $(U L)^{*}$. Deduce that $p$ th powers vanish in the cohomology algebra $H^{*}\left((U L)^{*}, \delta^{*}\right)$, which is the Hopf algebra dual of $H_{*} U(L, \delta)$. Thus $H_{*} U(L, \delta)$ is primitively generated.

\section{REFERENCES}

1. J. F. Adams, On the cobar construction, Proc. Nat. Acad. Sci. U.S.A. 42 (1956), 409-412.

2. J. F. Adams and P. J. Hilton, On the chain algebra of a loop space, Comment. Math. Helv. 30 (1955), 305-330.

3. D. J. Anick, A model of Adams-Hilton type for fiber squares, Illinois J. Math. 29 (1985), 463502.

4. M. Aubry and J.-M. Lemaire, Homotopies d'algèbres de Lie et de leurs algèbres enveloppantes, Algebraic Topology-Rational Homotopy, Lecture Notes in Math., no. 1318, Springer-Verlag, Berlin and New York, 1988, 26-30.

5. H. J. Baues, Algebraic homotopy, Cambridge Stud. Adv. Math., no. 15, Cambridge Univ. Press, Cambridge, 1989.

6. H. J. Baues, S. Halperin, and J.-M. Lemaire, The uniqueness of rational homotopy (in preparation).

7. H. J. Baues and J. M. Lemaire, Minimal models in homotopy theory, Math. Ann. 225 (1977), 219-242.

8. F. R. Cohen, J. C. Moore, and J. A. Neisendorfer, Torsion in homotopy groups, Ann. of Math. (2) 109 (1979), 121-168.

9. C. A. McGibbon and C. Wilkerson, Loop spaces of finite complexes at large primes, Proc. Amer. Math. Soc. 96 (1986), 698-702.

10. J. Milnor and J. C. Moore, On the structure of Hopf algebras, Ann. of Math. (2) 81 (1965), 211-264.

11. H. Munkholm, DGA algebras as a Quillen model category, J. Pure Appl. Algebra 13 (1978), 221-232.

12. D. G. Quillen, Homotopical algebra, Lecture Notes in Math., no. 43, Springer-Verlag, Berlin and New York, 1967.

13. __ Rational homotopy theory, Ann. of Math. (2) 90 (1969), 205-295.

14. D. Sullivan, Infinitesimal computations in topology, Inst. Hautes Études Sci. Publ. Math. 47 (1978), 269-331.

15. D. Tanré, Homotopie rationnelle: Modèles de Chen, Quillen, Sullivan, Lecture Notes in Math., no. 1025, Springer-Verlag, Berlin and New York, 1983.

ABSTRACT. Let $(A, d)$ denote a free $r$-reduced differential graded $R$-algebra, where $R$ is a commutative ring containing $n^{-1}$ for $1 \leq n<p$. Suppose a "diagonal" $\psi: A \rightarrow A \otimes A$ exists which satisfies the Hopf algebra axioms, including cocommutativity and coassociativity, up to homotopy. We show that $(A, d)$ must equal $U(L, \delta)$ for some free differential graded Lie algebra $(L, \delta)$ 
if $A$ is generated as an $R$-algebra in dimensions below $r p$. As a consequence, the rational singular chain complex on a topological monoid is seen to be the enveloping algebra of a Lie algebra. We also deduce, for an $r$-connected $\mathrm{CW}$ complex $X$ of dimension $\leq r p$, that the Adams-Hilton model over $R$ is an enveloping algebra and that $p$ th powers vanish in $\widetilde{H}^{*}\left(\Omega X ; \mathbf{Z}_{p}\right)$.

Department of Mathematics, Massachusetts institute of Technology, Cambridge, MASSACHUSETTS 02139 